\title{
Validação de metodologia QuEChERS-acetato para a análise de multirresíduo de agrotóxicos em amostras de soja e de extrato solúvel de soja utilizando cromatografia líquida de alta eficiência acoplada à espectrometria de massas sequencial
}

\section{Validation of acetateQuEChERS methodologyfor pesticides multiresidue analysis in soybean samples and soybean-soluble extract by means of high-performance liquid chromatography coupled to tandem mass spectrometry}

RIALA6/1588

Adherlene Vieira GOUVÊA ${ }^{1 *}$, Maria Helena Wohlers Morelli CARDOSO ${ }^{1}$, Lucia Helena Pinto BASTOS ${ }^{1}$, Cristiane BARATA SILVA $^{1}$, Nina Daddario ORTIZ ${ }^{2}$, Armi Wanderley da NÓBREGA ${ }^{1}$, Silvana do Couto JACOB ${ }^{1}$

${ }^{*}$ Endereço para correspondência: ${ }^{1}$ Laboratório de Alimentos e Contaminantes, Instituto Nacional de Controle da Qualidade em Saúde (INCQS), Fundação Oswaldo Cruz (FIOCRUZ). Av. Brasil, 4365 - Manguinhos, Rio de Janeiro, RJ, Brasil, 21040900. E-mail: adherlene.gouvea@incqs.fiocruz.br, adherlene@gmail.com.

${ }^{2}$ Unidade Proteômica, Departamento de Bioquímica, Instituto de Química (IQ), Universidade Federal do Rio de Janeiro (UFRJ) Recebido: 15.03.2013 - Aceito para publicação: 12.03.2014

\section{RESUMO}

Com o aumento da utilização da soja e seus derivados na alimentação humana torna-se importante a avaliação da possibilidade de contaminação desses produtos por resíduos de agrotóxicos. Neste contexto, este estudo efetuou a validação da metodologia QuEChERS-acetato, para análise de 144 resíduos de agrotóxicos em soja e no extrato solúvel de soja por meio de cromatografia líquida acoplada à espectrometria de massas sequencial. Os parâmetros avaliados foram: seletividade (efeito matriz nos dois tipos de amostras), linearidade (faixa de trabalho, significância da regressão e homogeneidade dos resíduos da regressão), exatidão (recuperação), precisão (repetitividade) e limite de detecção e de quantificação. As curvas analíticas apresentaram $\mathrm{R}^{2} \geq 0,95$ e $\mathrm{r} \geq 0,98$ na faixa de trabalho $\left(0,002\right.$ a $\left.0,200 \mu \mathrm{g} \cdot \mathrm{mL}^{-1}\right)$. A exatidão e precisão em dois níveis de fortificação das duas matrizes apresentaram valores de 70 a $120 \%$ de recuperação e $\mathrm{CV}(\%) \leq 20 \%$, respectivamente. O limite de detecção e de quantificação apresentou resultados satisfatórios. A metodologia validada possibilitou a determinação de 122 substâncias na matriz soja e 124 na matriz de extrato solúvel de soja.

Palavras-chave. soja, agrotóxicos, estudos de validação, cromatografia líquida, espectrometria de massas.

\begin{abstract}
The possibility of occurring the soy and soy derivatives samples contamination with pesticide residues has become significant due to the increased use of these products in human nourishment. In this context, the present study aimed at validating the acetate QuEChERS multiresidue methodology for analyzing 144 pesticide residues in soybean samples and soybean soluble extract using high-performance liquid chromatography coupled to tandem mass spectrometry. The parameters evaluated were: selectivity (matrix effect in the two sample types), linearity (working range, regression significance, homogeneity of the regression residues), accuracy (recovery), precision (repeatability) and limits of detection and quantification. The analytical curves presented $\mathrm{R}^{2} \geq 0.95$ and $\mathrm{r} \geq 0.98$ in the working range $(0.002$ to 0.200 $\left.\mu \mathrm{g} \cdot \mathrm{mL}^{-1}\right)$. The accuracy and precision evaluated at two levels of fortification of two matrices presented values of recovery in the $70-120 \%$ range and CV $(\%) \leq 20 \%$, respectively. The limits of detection and quantification showed satisfactory results. The validated methodology allowed to perform the determination of 122 substances in the matrix soybean and 124 in the soluble extract of soybean.

Keywords. soybeans, pesticides, validation studies, chromatography, liquid, mass spectrometry.
\end{abstract}




\section{INTRODUÇÃO}

$\mathrm{O}$ uso indiscriminado dos agrotóxicos em desrespeito às indicações agronômicas acarreta a presença de resíduos de agrotóxicos nos alimentos, em quantidades superiores àquelas estabelecidas na legislação, podendo expor a população a possíveis agravos à saúde.

Quando são utilizados agrotóxicos de uso não autorizado, a exposição da população que consome esses alimentos contaminados é de maior gravidade, visto que não existem estudos toxicológicos que possibilitem estabelecer, em âmbito nacional, limites seguros de resíduos que não causem danos aos consumidores desses alimentos.

A soja é a cultura agrícola que mais cresceu nas últimas três décadas e corresponde a $49 \%$ da área plantada em grãos do país. O cultivo é feito especialmente nas regiões centro-oeste e sul do país ${ }^{1}$. Dados da safra de 2010/2011 indicam que o Brasil é o segundo maior produtor mundial de soja, atrás apenas dos Estados Unidos ${ }^{2,3}$.

Há evidências de que nos últimos anos a inclusão de produtos à base de soja vem intensificando-se na alimentação humana, pois o grão é um dos alimentos mais completos em relação às proteínas ${ }^{4}$. A indicação de uso de alimentos à base de soja também inclui pessoas com histórico familiar de osteoporose e câncer de próstata ${ }^{5}$.

Para a cultura da soja, atualmente, são permitidos no Brasil cerca de cento e cinquenta e cinco agrotóxicos, treze produtos biológicos, quatro ferormônios sintéticos, quatro reguladores de crescimento e cinco compostos inorgânicos. Dentre os agrotóxicos permitidos, vinte e nove são da classificação toxicológica $I$, trinta e um da classificação toxicológica II, sessenta e nove da classificação toxicológica III e vinte e seis da classificação toxicológica $\mathrm{IV}^{6}$.

O avanço da tecnologia impacta no aumento da produção e do consumo de soja e seus derivados, sendo necessário o desenvolvimento de métodos para controle destes produtos visando verificar o cumprimento da legislação a fim de prever o aparecimento de danos à saúde.

As análises de resíduos de agrotóxicos em alimentos são muito dispendiosas principalmente no que se refere à utilização de materiais de referência para a quantificação e identificação dessas substâncias devido ao elevado número de agrotóxicos que devem ser investigados. Outro fator determinante para o elevado custo dessa análise é a necessidade de utilização de equipamentos sofisticados para atender aos limites requeridos e treinamento de técnicos especializados ${ }^{7}$.
Dificuldades existem no que se refere à quantidade de substâncias permitidas para o uso e controle de pragas na produção de alimentos, a diversidade das características físico-químicas dessas substâncias e o uso indevido de agrotóxicos não permitidos na legislação vigente.

Porém, são os resultados dessas análises o referencial para se avaliar os níveis de resíduos de agrotóxicos que chegam à mesa do consumidor e, também, serve como subsídio técnico-científico para as ações de vigilância no sentido de prevenir agravos à saúde da população devido à exposição a estas substâncias químicas.

Neste desafio os métodos de análise adequados são denominados multirresiduais que englobam a quantificação destes agrotóxicos em uma mesma corrida cromatográfica.

Pizzutti et $\mathrm{al}^{8}$ realizou seu estudo com cento e sessenta e nove agrotóxicos em grãos de soja utilizando o método multirresíduo Luke modificado o qual consiste em uma extração com acetona, éter de petróleo e diclorometano. Uma desvantagem do método é a utilização de grande quantidade de solvente.

O método multirresíduo denominado QuEChERS ("Quick, Easy, Cheap, Effective, Rugged, Safe") desenvolvido em 2003 por Anastassiades et $\mathrm{al}^{9}$ tem como vantagens o fato de ser rápido, fácil, econômico, efetivo, robusto e seguro. Diversas modificações foram realizadas ao longo do tempo nesse método, com o objetivo de aumentar a aplicação para diversas matrizes, com altos percentuais de recuperação (>85 \%) para substâncias de diferentes polaridades e volatilidades além da redução da quantidade de solventes orgânicos utilizados ${ }^{10}$.

Pizzutti et al $^{11}$ também realizou um estudo comparativo desses cento e sessenta e nove agrotóxicos analisados com o método multirresíduo Luke modificado $^{8}$ e com uma diferente versão do método QuEChERS-acetato utilizando acetonitrila acidificada, sulfato de magnésio anidro e acetato de sódio anidro, sem a etapa de "clean up". Ambos os métodos demonstraram ser adequados para a utilização na matriz grãos de soja respeitando os critérios internacionais de qualidade. Os resultados encontrados mostraram um melhor desempenho do método QuEChERS-acetato nos critérios de exatidão e precisão em relação ao método Luke modificado.

No entanto, a etapa de "clean-up" é essencial para resultados satisfatórios, aumentando a eficiência do método em relação ao número de substâncias com 
resultados adequados para os critérios de exatidão e precisão, promovendo robustez e confiabilidade. Outro fator determinante na utilização dessa etapa consiste na manutenção das condições de uso do equipamento, pois componentes não voláteis da matriz podem ficar aderidos no conjunto injetor-insersor e também na coluna cromatográfica alterando a resposta do sistema e aumentando a frequência de manutenções necessárias ${ }^{10}$.

O desenvolvimento e aprimoramento do QuEChERS para diferentes matrizes foi um marco na análise de resíduos de agrotóxicos pois agregou rapidez, facilidade de execução, economia, precisão e exatidão.

Informações de identificação, quantificação e confirmação da presença dos resíduos dessas substâncias são importantes para avaliação do risco real pelo qual a população consumidora está exposta. $\mathrm{O}$ avanço da cromatografia líquida associada à espectrometria de massas sequencial subsidia esta necessidade elencandose como a principal técnica utilizada para a análise de resíduos de agrotóxicos com o maior número de substâncias monitoradas.

Para a utilização de métodos não oficiais, ou seja, que não possuem uma comprovação de atendimento a requisitos para uma aplicação específica, é necessária a submissão do método a um processo de validação. Este processo é a comprovação, através de evidência objetiva, que os requisitos para uma aplicação ou uso específico pretendido são atendidos ${ }^{12}$.

A validação de métodos tem por objetivo confirmá-los como adequados para o uso pretendido. Este procedimento se aplica a métodos não normalizados, criados ou desenvolvidos pelo próprio laboratório, normalizados usados fora dos escopos para os quais foram concebidos ampliações e modificações de métodos normalizados ${ }^{13}$.

Os estudos de validação devem ser representativos e adequados para as substâncias de interesse, nos níveis delimitados pela legislação vigente, além de apresentar parâmetros aceitáveis de desempenho analítico que sejam capazes de garantir a confiabilidade dos resultados.

A seletividade, uma das etapas da validação, é a avaliação da presença de interferentes das substâncias de interesse na matriz da amostra a ser utilizada na validação. Os experimentos de avaliação de seletividade incluem ensaios com amostras com e sem o analito pesquisado ${ }^{13}$. Esta etapa do processo deve ser assegurada para não comprometer os outros parâmetros da validação a serem avaliados.
A construção de curvas analíticas é uma prática comum em laboratório, sendo este passo de extrema importância para a obtenção de resultados precisos e com boa exatidão ${ }^{14}$. De acordo com o trabalho a ser desenvolvido, uma faixa de concentração do analito na qual o método pode ser aplicado deve ser definida, sendo denominada faixa de trabalho. Essa faixa deve cobrir os níveis de concentração para os quais o ensaio será aplicado. Nas análises de multirresíduos, como no caso dos agrotóxicos, o valor da faixa de trabalho deve abranger o menor e o maior LMR permitido para o grupo de substâncias estudadas.

Para a quantificação do analito na amostra é necessário que se conheça a dependência entre a resposta medida e a concentração do analito. A linearidade corresponde à capacidade do método em fornecer resultados diretamente proporcionais à concentração da substância em análise ${ }^{15}$. Levando-se em consideração que a maioria dos métodos analíticos utiliza relações lineares para quantificação analítica, o exame de uma função de calibração para a linearidade é uma figura de desempenho importante na validação de um método analítico ${ }^{16}$. A faixa de trabalho, com uma resposta linear é denominada faixa linear.

Bazilio et $\mathrm{al}^{14}$ apresentam uma planilha eletrônica, intitulada "Planilha para Avaliação de Premissas" para cálculo e avaliação da linearidade de curvas analíticas. Os parâmetros de regressão podem ser estimados com o auxílio desta planilha. Outros resultados apresentados são a avaliação da homogeneidade dos resíduos da regressão, $r, \mathrm{R}^{2}$ e a significância da regressão.

A matrizda amostra pode conter componentes que interferem no desempenho da medição, podendo haver acréscimo ou redução do sinal, sendo que a magnitude do efeito também pode depender da concentração. Se o efeito matriz for significativo, a linearidade, a tendência e a precisão estarão seriamente comprometidas ${ }^{13}$.

O processo de preparação dos padrões de calibração pode ser simplificado se os mesmos forem preparados com soluções simples do analito. Porém, é necessário que seja avaliada uma possível incompatibilidade da matriz analisada antes da sua utilização no preparo dos padrões ${ }^{17}$.

$\mathrm{O}$ efeito matriz pode variar de ocorrência e intensidade, porém algumas técnicas apresentam uma maior magnitude do efeito. Desta forma, se a técnica utilizada não é inerte ao efeito matriz é necessária a avaliação no processo de validação para a garantia de um 
resultado fidedigno ${ }^{18}$.

Souza ${ }^{19}$ descreve um procedimento analítico para avaliação do efeito matriz, na qual uma curva analítica preparada, em solvente, é comparada através do teste $t$ com uma curva preparada na presença da matriz da amostra. Quando as curvas analíticas preparadas não diferem estatisticamente entre si considera-se que o efeito matriz não é significativo.

O limite de detecção (LD) representa a menor concentração do analito que pode ser detectada e o limite de quantificação (LQ) representa a menor concentração do analito que pode ser quantificada. Um dos métodos de determinação destes limites é o método da relação sinal/ruído (S/R). O LD é a concentração que produziu um sinal 3 vezes maior do que o ruído da linha de base; e o LQ é a concentração que produziu um sinal 10 vezes maior do que o ruído da linha de base ${ }^{20}$. Os limites de detecção e quantificação adequados para a análise multirresíduos de agrotóxicos têm valores inferiores aos LMR das substâncias estudadas.

Os estudos dos limites de detecção e quantificação feitos através da adição da solução de interesse na amostra branco avaliada no estudo de seletividade antes do processo de extração são denominados fortificação da amostra.

Outro parâmetro avaliado no processo de validação é a exatidão que é o grau de concordância existente entre o valor encontrado e o valor aceito como verdadeiro, sendo avaliada numericamente através da tendência. Esta é uma combinação de componentes de erros aleatórios e sistemáticos podendo ser expressa como a recuperação analítica. Um dos processos utilizados para avaliação da tendência é a realização de ensaios de recuperação e os valores aceitáveis de recuperação estabelecidos pelo SANCO ${ }^{18}$ são 70-120 \%.

A precisão representa a dispersão de resultados entre ensaios independentes de uma mesma amostra, amostras semelhantes ou padrões em determinadas condições ${ }^{15}$, pode ser expressa de três formas: precisão intermediária, reprodutibilidade e repetitividade sendo que esta última é avaliada pelo coeficiente de variação $(\mathrm{CV}(\%))$ e/ou estimativa do desvio padrão relativo $(\mathrm{DPR}(\%))$.

Os ensaios de recuperação para avaliação da exatidão e precisão também são feitos através da fortificação de amostras em diferentes níveis de concentrações e replicatas de análise.

As condições de repetitividade podem ser caracterizadas com: um mesmo procedimento de medição, um mesmo observador, um mesmo instrumento usado sob mesmas condições, um mesmo local e repetições no menor espaço de tempo possível ${ }^{13}$. Os valores aceitáveis de DPR(\%) ou CV(\%) definidos pelo $\mathrm{SANCO}^{18}$ são $\leq 20 \%$.

Em vista do aumento da utilização da soja e seus derivados na alimentação humana com ênfase em populações específicas como crianças, doentes, homens, mulheres e idosos, torna-se importante a avaliação destes alimentos quanto à contaminação de resíduos de agrotóxicos. Esta importância se refere à garantia do direito do consumidor às informações confiáveis a cerca dos alimentos comercializados.

Nesse contexto, o desenvolvimento desse estudo teve por finalidade validar o método QuEChERSacetato $^{9,21}$ para a análise de cento e quarenta e quatro resíduos de agrotóxicos em soja e no extrato solúvel de soja utilizando a técnica de cromatografia líquida de alta eficiência acoplada à espectrometria de massas sequencial - CLAE-EM/EM (High Performance Liquid Chromatography-Mass Spectrometry/ Mass Spectrometry - HPLC-MS/MS). O trabalho permitiu fornecer subsídios para avaliação da situação atual do consumo desses alimentos, disponíveis no mercado nacional em relação à presença destes resíduos.

\section{MATERIAL E MÉTODOS}

\section{Reagentes, solventes e soluções}

Os reagentes e solventes utilizados para o desenvolvimento do trabalho foram o acetato de etila grau resíduo, o acetato de sódio grau para análise, a acetona grau resíduo e o ácido acético glacial grau cromatografia líquida, fornecidos pela TEDIA (EUA), a acetonitrila grau cromatografia líquida, o formato de amônio grau para análise e o sulfato de magnésio anidro grau para análise, fornecidos pela Sigma-Aldrich (EUA), a água tipo I, o detergente Extran ${ }^{\circledR}$ alcalino grau para análise, fornecido pela Merck (EUA), o metanol grau espectrometria de massas, fornecido pela J. T. Baker (EUA), o sorvente amina primária- secundária ("primary secondary amine" - PSA) grau para análise, fornecida pela Varian (EUA).

As soluções estoques dos agrotóxicos foram preparadas a partir dos padrões de agrotóxicos adquiridos do Dr. Ehrenstofer (Alemanha), com certificado de análise e grau de pureza superior a $95 \%$, diluídos em 
acetato de etila e metanol dependendo da solubilidade de cada substância. As soluções estoque foram preparadas na concentração nominal de $100 \mu \mathrm{g} \cdot \mathrm{mL}^{-1}$, de acordo com a pureza definida no seu certificado e a aplicação pretendida. A partir dessas soluções foi preparada uma mistura na concentração nominal de $1 \mu \mathrm{g} \cdot \mathrm{mL}^{-1}$. Com essa mistura foram preparadas todas as soluções de trabalho nas seguintes concentrações nominais: 0,$002 ; 0,004$; 0,$008 ; 0,01 ; 0,02 ; 0,04 ; 0,08 ; 0,1$ e $0,2 \mu \mathrm{g} \cdot \mathrm{mL}^{-1}$.

\section{Equipamentos e condições operacionais}

Foi utilizado o cromatógrafo líquido de ultra eficiência (Waters, EUA) modelo ACQUITY UPLC ${ }^{\mathrm{TM}}$ equipado com um sistema binário de bombas, injetor automático, degaseificador e forno para a coluna. A coluna utilizada para a separação cromatográfica foi de fase reversa Alltima ${ }^{\mathrm{TM}} \mathrm{C}_{18}$ com $5 \mu \mathrm{m}$ de tamanho de partícula, $3.2 \mathrm{~mm}$ de diâmetro interno e $150 \mathrm{~mm}$ de comprimento (Grace, EUA). A pré-coluna utilizada foi o cartucho SecurityGuard $\mathrm{C}_{18}$ com $4 \mathrm{~mm}$ de diâmetro interno e $3 \mathrm{~mm}$ de comprimento (Phenomenex, EUA). $O$ detector acoplado foi o detector de massas sequencial (Waters, EUA) modelo Quattro Premier $\mathrm{XE}^{\mathrm{TM}}$ equipado com uma fonte de ionização Electrospray Ionization
(ESI) (Z-Spray ${ }^{\mathrm{TM}}$ ) operando no modo positivo e estação de trabalho MassLynx ${ }^{\mathrm{TM}}$ Versão 4.1.

As condições operacionais utilizadas no cromatógrafo líquido foram o volume de injeção de 5 $\mu \mathrm{L}$, a vazão da fase móvel constante de $0,3 \mathrm{~mL} \cdot \mathrm{min}^{-1}$, a temperatura do forno da coluna constante de $40{ }^{\circ} \mathrm{C}$, a temperatura do amostrador constante de $8^{\circ} \mathrm{C}$ e o tempo total de corrida de $38 \mathrm{~min}$. Na eluição por gradiente, as fases móveis utilizadas no estudo foram a fase denominada A: formato de amônio $5 \times 10^{-3}$ mol. $\mathrm{L}^{-1} \mathrm{com}$ $10 \%$ de metanol e a fase denominada B: metanol.

As condições operacionais utilizadas no espectrômetro de massas sequencial foram a voltagem do capilar de $0,98 \mathrm{kV}$, a temperatura da fonte $\mathrm{ESI}^{+} \mathrm{em}$ $120^{\circ} \mathrm{C}$, a temperatura do gás de dessolvatação $\left(\mathrm{N}_{2}\right)$ de $400{ }^{\circ} \mathrm{C}$, o fluxo do gás do cone $\left(\mathrm{N}_{2}\right)$ de $50 \mathrm{~L} . \mathrm{h}^{-1}$, o fluxo do gás de dessolvatação $\left(\mathrm{N}_{2}\right)$ de $800 \mathrm{~L} \cdot \mathrm{h}^{-1}$ e a pressão do gás de colisão (Argônio) de $3,5 \times 10^{-3}$ mbar.

$\mathrm{Na}$ Tabela 1 estão relacionadas as transições selecionadas para a quantificação e confirmação de cada substância selecionada para o estudo de acordo com as referências consultadas ${ }^{22-39}$. A letra " $\mathrm{s}$ " ao lado do nome das substâncias indica o uso permitido para a cultura da soja.

Outros equipamentos utilizados para a realização

Tabela 1. Condições analíticas para detecção e quantificação das substâncias selecionadas para o estudo

\begin{tabular}{|c|c|c|c|c|c|c|c|c|c|c|c|c|}
\hline \multirow[t]{2}{*}{ No } & \multirow{2}{*}{$\begin{array}{l}\text { Substância } \\
\text { 3-Hidroxi-Carbo- } \\
\text { furano }\end{array}$} & \multicolumn{2}{|c|}{$\begin{array}{c}\text { Transição } \\
\text { Quantificação } \\
\text { MRM }\end{array}$} & \multicolumn{2}{|c|}{$\begin{array}{c}\text { Transição } \\
\text { Confirmação } \\
\text { MRM }\end{array}$} & \multicolumn{2}{|c|}{$\begin{array}{l}\text { Espécie } \\
\text { do Î́n } \\
\text { Precursor }\end{array}$} & \multirow{2}{*}{$\begin{array}{c}\begin{array}{c}\text { Espécie } \\
\text { do Íon } \\
\text { Precursor }\end{array} \\
{[\mathrm{M}+\mathrm{H}]^{+}}\end{array}$} & \multirow{2}{*}{$\begin{array}{c}\begin{array}{c}\text { Voltagem } \\
\text { do Cone } \\
(\mathrm{V})\end{array} \\
18\end{array}$} & \multirow{2}{*}{$\begin{array}{c}\begin{array}{c}\text { Energia de } \\
\text { Colisão } \\
\text { Quant. } \\
(\mathrm{eV})\end{array} \\
15\end{array}$} & \multirow{2}{*}{$\begin{array}{c}\begin{array}{c}\text { Energia de } \\
\text { Colisão } \\
\text { Confirm. } \\
(\mathrm{eV})\end{array} \\
10\end{array}$} & \multirow{2}{*}{$\begin{array}{c}\begin{array}{c}\mathrm{Tr} \\
(\mathrm{min})\end{array} \\
8.40\end{array}$} \\
\hline & & 237,9 & $>$ & 162,8 & 237,9 & $>$ & 180,8 & & & & & \\
\hline 2 & Abamectina (s) & 890,5 & $>$ & 567,1 & 890,5 & $>$ & 305,0 & {$[\mathrm{M}+\mathrm{NH} 4]^{+}$} & 20 & 20 & 15 & 19,26 \\
\hline 4 & Acetamiprido (s) & 223,1 & $>$ & 125,9 & 223,1 & $>$ & 90,0 & {$[\mathrm{M}+\mathrm{H}]^{+}$} & 35 & 35 & 22 & 8,66 \\
\hline 5 & Aldicarbe & 190,5 & $>$ & 115,6 & 190,5 & $>$ & 88,7 & {$[\mathrm{M}+\mathrm{H}]^{+}$} & 12 & 14 & 5 & 10,56 \\
\hline 6 & Aldicarbe Sulfona & 223,1 & $>$ & 76,0 & 223,1 & $>$ & 86,0 & {$[\mathrm{M}+\mathrm{H}]^{+}$} & 25 & 10 & 14 & 5,18 \\
\hline 7 & Aldicarbe Sulfóxido & 207,0 & $>$ & 88,9 & 207,0 & $>$ & 131,9 & {$[\mathrm{M}+\mathrm{H}]^{+}$} & 20 & 14 & 10 & 4,69 \\
\hline 11 & Azinfós-Etílico & 345,5 & $>$ & 131,8 & 345,5 & $>$ & 159,8 & {$[\mathrm{M}+\mathrm{H}]^{+}$} & 15 & 18 & 10 & 15,80 \\
\hline 12 & Azinfós-Metílico & 318,0 & $>$ & 131,9 & 318,0 & $>$ & 104,2 & {$[\mathrm{M}+\mathrm{H}]^{+}$} & 15 & 24 & 16 & 14,25 \\
\hline 13 & Azoxistrobina (s) & 404,1 & $>$ & 372,0 & 404,1 & $>$ & 328,9 & {$[\mathrm{M}+\mathrm{H}]^{+}$} & 15 & 30 & 16 & 14,11 \\
\hline 14 & Benalaxil & 325,9 & $>$ & 147,9 & 325,9 & $>$ & 293,9 & {$[\mathrm{M}+\mathrm{H}]^{+}$} & 20 & 20 & 20 & 16,56 \\
\hline 15 & Boscalida & 343,0 & $>$ & 307,0 & 343,0 & $>$ & 271,2 & {$[\mathrm{M}+\mathrm{H}]+$} & 30 & 30 & 20 & 14,69 \\
\hline 16 & Bromuconazol (s) & 376,0 & $>$ & 70,1 & 376,0 & $>$ & 158,9 & {$[\mathrm{M}+\mathrm{H}]+$} & 35 & 25 & 35 & 15,47 \\
\hline
\end{tabular}


Gouvêa AV, Cardoso MHWM, Bastos LHP, Silva CB, Ortiz ND, Nóbrega AWE, et al. Validação de metodologia QuEChERS-acetato para a análise de multirresíduo de agrotóxicos em amostras de soja e de extrato solúvel de soja utilizando cromatografia líquida de alta eficiência acoplada à espectrometria de massas sequencial. Rev Inst Adolfo Lutz. São Paulo, 2014; 73(1):40-58.

\begin{tabular}{|c|c|c|c|c|c|c|c|c|c|c|c|c|}
\hline \multicolumn{13}{|c|}{ Cont. Tabela 1} \\
\hline \multirow{2}{*}{$\begin{array}{l}\text { No } \\
17\end{array}$} & \multirow{2}{*}{$\begin{array}{l}\text { Substância } \\
\text { Bupirimate }\end{array}$} & \multicolumn{2}{|c|}{$\begin{array}{c}\text { Transição } \\
\text { Quantificação } \\
\text { MRM }\end{array}$} & \multicolumn{2}{|c|}{$\begin{array}{c}\text { Transição } \\
\text { Confirmação } \\
\text { MRM }\end{array}$} & \multicolumn{2}{|c|}{$\begin{array}{l}\text { Espécie } \\
\text { do Î́on } \\
\text { Precursor }\end{array}$} & \multirow{2}{*}{$\begin{array}{c}\begin{array}{c}\text { Espécie } \\
\text { do Íon } \\
\text { Precursor }\end{array} \\
{[\mathrm{M}+\mathrm{H}]^{+}}\end{array}$} & \multirow{2}{*}{$\begin{array}{c}\begin{array}{c}\text { Voltagem } \\
\text { do Cone } \\
(\mathrm{V})\end{array} \\
35\end{array}$} & \multirow{2}{*}{$\begin{array}{c}\begin{array}{c}\text { Energia de } \\
\text { Colisão } \\
\text { Quant. } \\
(\mathrm{eV})\end{array} \\
25\end{array}$} & \multirow{2}{*}{$\begin{array}{c}\begin{array}{c}\text { Energia de } \\
\text { Colisão } \\
\text { Confirm. } \\
(\mathrm{eV})\end{array} \\
20\end{array}$} & \multirow{2}{*}{$\begin{array}{c}\begin{array}{c}\mathrm{Tr} \\
(\mathrm{min})\end{array} \\
16,18\end{array}$} \\
\hline & & 316,8 & $>$ & 107,6 & 316,8 & $>$ & 271,7 & & & & & \\
\hline 18 & Buprofenzina (s) & 306,1 & $>$ & 201,0 & 306,1 & $>$ & 115,9 & {$[\mathrm{M}+\mathrm{H}]^{+}$} & 20 & 16 & 12 & 17,95 \\
\hline 19 & $\begin{array}{l}\text { Butocarboxim } \\
\text { Sulfóxido }\end{array}$ & 207,0 & $>$ & 88,0 & 207,0 & $>$ & 75,0 & {$[\mathrm{M}+\mathrm{H}]^{+}$} & 20 & 10 & 10 & 4,42 \\
\hline 20 & Cadusafós & 271,4 & $>$ & 159,0 & 271,4 & $>$ & 215,0 & {$[\mathrm{M}+\mathrm{H}]^{+}$} & 25 & 15 & 10 & 17,27 \\
\hline 21 & Carbaril & 219,0 & $>$ & 144,9 & 219,0 & $>$ & 126,9 & {$[\mathrm{M}+\mathrm{NH} 4]^{+}$} & 10 & 35 & 16 & 12,45 \\
\hline 22 & Carbendazim (s) & 192,0 & $>$ & 159,9 & 192,0 & $>$ & 132,0 & {$[\mathrm{M}+\mathrm{H}]^{+}$} & 25 & 30 & 16 & 10,23 \\
\hline 23 & Carbofurano & 222,1 & $>$ & 122,9 & 222,1 & $>$ & 164,9 & {$[\mathrm{M}+\mathrm{H}]^{+}$} & 25 & 25 & 12 & 11,82 \\
\hline 24 & Carbossulfano (s) & 381,0 & $>$ & 160,0 & 381,0 & $>$ & 118,0 & {$[\mathrm{M}+\mathrm{H}]^{+}$} & 30 & 20 & 15 & 20,22 \\
\hline 25 & Ciazofamida & 324,6 & $>$ & 107,6 & 324,6 & $>$ & 260,7 & {$[\mathrm{M}+\mathrm{H}]^{+}$} & 20 & 15 & 10 & 15,71 \\
\hline 26 & Cimoxanil & 199,0 & $>$ & 127,9 & 199,0 & $>$ & 110,9 & {$[\mathrm{M}+\mathrm{H}]^{+}$} & 20 & 18 & 10 & 9,51 \\
\hline 27 & Ciproconazol (s) & 292,1 & $>$ & 70,1 & 292,1 & $>$ & 124,9 & {$[\mathrm{M}+\mathrm{H}]^{+}$} & 25 & 18 & 30 & 15,29 \\
\hline 28 & Ciprodinil & 226,1 & $>$ & 92,9 & 226,1 & $>$ & 107,9 & {$[\mathrm{M}+\mathrm{H}]^{+}$} & 45 & 35 & 25 & 17,08 \\
\hline 29 & Ciromazina & 166,8 & $>$ & 60,0 & 166,8 & $>$ & 124,8 & {$[\mathrm{M}+\mathrm{H}]^{+}$} & 30 & 18 & 18 & 5,14 \\
\hline 30 & Clofentezina & 303,0 & $>$ & 137,9 & 303,0 & $>$ & 101,9 & {$[\mathrm{M}+\mathrm{H}]^{+}$} & 20 & 35 & 14 & 17,27 \\
\hline 31 & Clorbromuron & 294,5 & $>$ & 205,6 & 294,5 & $>$ & 181,6 & {$[\mathrm{M}+\mathrm{H}]^{+}$} & 25 & 20 & 20 & 15,18 \\
\hline 32 & Clorfenvinfós & 359,0 & $>$ & 98,8 & 359,0 & $>$ & 126,9 & {$[\mathrm{M}+\mathrm{H}]^{+}$} & 25 & 24 & 22 & 16,56 \\
\hline 33 & Clorpirifós (s) & 349,5 & $>$ & 197,7 & 349,5 & $>$ & 96,7 & {$[\mathrm{M}+\mathrm{H}]^{+}$} & 20 & 30 & 21 & 18,52 \\
\hline 34 & Clotianidina (s) & 250,0 & $>$ & 168,9 & 250,0 & $>$ & 131,8 & {$[\mathrm{M}+\mathrm{H}]^{+}$} & 20 & 14 & 14 & 7,96 \\
\hline 35 & Coumafós & 363,0 & $>$ & 307,0 & 363,0 & $>$ & 289,0 & {$[\mathrm{M}+\mathrm{H}]^{+}$} & 26 & 16 & 24 & 16,66 \\
\hline 36 & Cresoxim-Metílico (s) & 313,7 & $>$ & 266,7 & 313,7 & $>$ & 115,7 & {$[\mathrm{M}+\mathrm{H}]^{+}$} & 15 & 15 & 5 & 16,37 \\
\hline 37 & Demeton-S-Metílico & 230,7 & $>$ & 88,9 & 230,7 & $>$ & 61,1 & {$[\mathrm{M}+\mathrm{H}]^{+}$} & 12 & 30 & 10 & 12,11 \\
\hline 38 & Desmedifam & 318,2 & $>$ & 181,9 & 318,2 & $>$ & 135,9 & {$[\mathrm{M}+\mathrm{NH} 4]^{+}$} & 20 & 30 & 14 & 13.61 \\
\hline 39 & Diazinona & 305,1 & $>$ & 169,0 & 305,1 & $>$ & 96,9 & {$[\mathrm{M}+\mathrm{H}]^{+}$} & 25 & 35 & 22 & 16,75 \\
\hline 40 & Diclorvós & 220,9 & $>$ & 126,9 & 220,9 & $>$ & 108,8 & {$[\mathrm{M}+\mathrm{H}]^{+}$} & 25 & 18 & 18 & 11,85 \\
\hline 41 & Difenoconazol (s) & 406,1 & $>$ & 250,9 & 406,1 & $>$ & 187,8 & {$[\mathrm{M}+\mathrm{H}]^{+}$} & 35 & 40 & 25 & 17,16 \\
\hline 42 & Dimetoato & 230,0 & $>$ & 198,8 & 230,0 & $>$ & 124,8 & {$[\mathrm{M}+\mathrm{H}]^{+}$} & 20 & 22 & 10 & 8,78 \\
\hline 43 & Dimetomorfe & 388,1 & $>$ & 300,9 & 388,1 & $>$ & 165,0 & {$[\mathrm{M}+\mathrm{H}]^{+}$} & 35 & 30 & 20 & 14,93 \\
\hline 44 & Diniconazol & 326,1 & $>$ & 70,1 & 326,1 & $>$ & 158,9 & {$[\mathrm{M}+\mathrm{H}]^{+}$} & 35 & 25 & 30 & 17,19 \\
\hline 45 & Dissulfotom & 274,7 & $>$ & 61,0 & 274,7 & $>$ & 88,8 & {$[\mathrm{M}+\mathrm{H}]^{+}$} & 12 & 35 & 10 & 17,27 \\
\hline 46 & Diurom (s) & 233,0 & $>$ & 72,0 & 233,0 & $>$ & 159,9 & {$[\mathrm{M}+\mathrm{H}]^{+}$} & 25 & 18 & 25 & 13,92 \\
\hline 47 & Dodemorfe & 282,3 & $>$ & 115,9 & 282,3 & $>$ & 97,9 & {$[\mathrm{M}+\mathrm{H}]^{+}$} & 40 & 29 & 21 & 24.60 \\
\hline 48 & Epoxiconazol (s) & 330,1 & $>$ & 120,9 & 330,1 & $>$ & 122,9 & {$[\mathrm{M}+\mathrm{H}]^{+}$} & 35 & 25 & 20 & 15,80 \\
\hline \multirow{2}{*}{49} & Espinosade A (s) & 732,6 & $>$ & 142,0 & 732,6 & $>$ & 98,1 & {$[\mathrm{M}+\mathrm{H}]^{+}$} & 50 & 59 & 31 & 22,60 \\
\hline & Espinosade D (s) & 746,5 & $>$ & 142,0 & 746,5 & $>$ & 98,1 & {$[\mathrm{M}+\mathrm{H}]^{+}$} & 45 & 55 & 31 & 23,78 \\
\hline 50 & Etiofencarbe-Sulfona & 275,1 & $>$ & 106,9 & 275,1 & $>$ & 201,0 & {$[\mathrm{M}+\mathrm{NH} 4]^{+}$} & 15 & 22 & 10 & 7,17 \\
\hline 51 & Etiofencarbe-Sulfóxido & 242,1 & $>$ & 106,9 & 242,1 & $>$ & 184,9 & {$[\mathrm{M}+\mathrm{H}]^{+}$} & 15 & 25 & 10 & 7,35 \\
\hline 52 & Etiona & 384,6 & $>$ & 198,7 & 384,6 & $>$ & 142,7 & {$[\mathrm{M}+\mathrm{H}]^{+}$} & 20 & 25 & 10 & 18,01 \\
\hline 53 & Etiprole & 414,1 & $>$ & 350,9 & 414,1 & $>$ & 396,9 & {$[\mathrm{M}+\mathrm{NH} 4]^{ \pm}$} & 15 & 25 & 9 & 14,38 \\
\hline 54 & Etirimol & 210,1 & $>$ & 97,9 & 210,1 & $>$ & 140,0 & {$[\mathrm{M}+\mathrm{H}]^{+}$} & 40 & 25 & 22 & 13,87 \\
\hline
\end{tabular}


Gouvêa AV, Cardoso MHWM, Bastos LHP, Silva CB, Ortiz ND, Nóbrega AWE, et al. Validação de metodologia QuEChERS-acetato para a análise de multirresíduo de agrotóxicos em amostras de soja e de extrato solúvel de soja utilizando cromatografia líquida de alta eficiência acoplada à espectrometria de massas sequencial.

Rev Inst Adolfo Lutz. São Paulo, 2014; 73(1):40-58.

\begin{tabular}{|c|c|c|c|c|c|c|c|c|c|c|c|c|}
\hline \multirow{2}{*}{$\begin{array}{l}\text { No } \\
55\end{array}$} & \multirow{2}{*}{$\begin{array}{l}\text { Substância } \\
\text { Etoprofós }\end{array}$} & \multicolumn{2}{|c|}{$\begin{array}{c}\text { Transição } \\
\text { Quantificação } \\
\text { MRM }\end{array}$} & \multicolumn{2}{|c|}{$\begin{array}{c}\text { Transição } \\
\text { Confirmação } \\
\text { MRM }\end{array}$} & \multicolumn{2}{|c|}{$\begin{array}{l}\text { Espécie } \\
\text { do Íon } \\
\text { Precursor }\end{array}$} & \multirow{2}{*}{$\begin{array}{c}\begin{array}{c}\text { Espécie } \\
\text { do Îon } \\
\text { Precursor }\end{array} \\
{[\mathrm{M}+\mathrm{H}]^{+}}\end{array}$} & \multirow{2}{*}{$\begin{array}{c}\begin{array}{c}\text { Voltagem } \\
\text { do Cone } \\
(\mathrm{V})\end{array} \\
25\end{array}$} & \multirow{2}{*}{$\begin{array}{c}\begin{array}{c}\text { Energia de } \\
\text { Colisão } \\
\text { Quant. } \\
(\mathbf{e V})\end{array} \\
30\end{array}$} & \multirow{2}{*}{$\begin{array}{c}\begin{array}{c}\text { Energia de } \\
\text { Colisão } \\
\text { Confirm. } \\
(\mathbf{e V})\end{array} \\
20\end{array}$} & \multirow{2}{*}{$\begin{array}{c}\begin{array}{c}\mathrm{Tr} \\
(\mathrm{min})\end{array} \\
15,88\end{array}$} \\
\hline & & 243,0 & $>$ & 131,0 & 243,0 & $>$ & 97,0 & & & & & \\
\hline 56 & Etrinfós & 293,0 & $>$ & 265,0 & 293,0 & $>$ & 125,0 & {$[\mathrm{M}+\mathrm{H}]^{+}$} & 35 & 25 & 15 & 16,75 \\
\hline 57 & Famoxadona & 391,7 & $>$ & 330,8 & 391,7 & $>$ & 237,7 & {$[\mathrm{M}+\mathrm{NH} 4]^{+}$} & 15 & 18 & 10 & 16,28 \\
\hline 58 & Fenamidona & 312,1 & $>$ & 92,0 & 312,1 & $>$ & 236,1 & {$[\mathrm{M}+\mathrm{H}]^{+}$} & 25 & 25 & 14 & 14,57 \\
\hline 59 & Fenamifós & 304,1 & $>$ & 216,9 & 304,1 & $>$ & 201,9 & {$[\mathrm{M}+\mathrm{H}]^{+}$} & 30 & 35 & 24 & 15,91 \\
\hline 60 & Fenarimol (s) & 330,6 & $>$ & 267,6 & 330,6 & $>$ & 80,8 & {$[\mathrm{M}+\mathrm{H}]^{+}$} & 30 & 25 & 25 & 15,63 \\
\hline 61 & Fenazaquina & 307,2 & $>$ & 57,2 & 307,2 & $>$ & 161,0 & {$[\mathrm{M}+\mathrm{H}]^{+}$} & 30 & 25 & 19 & 20,87 \\
\hline 62 & Fenbuconazole & 336,8 & $>$ & 69,8 & 336,8 & $>$ & 124,7 & {$[\mathrm{M}+\mathrm{H}]^{+}$} & 30 & 20 & 25 & 15,71 \\
\hline 63 & Fenhexamide & 301,9 & $>$ & 96,8 & 301,9 & $>$ & 55,0 & {$[\mathrm{M}+\mathrm{H}]^{+}$} & 35 & 40 & 25 & 15,47 \\
\hline 64 & Fenoxicarbe & 302,1 & $>$ & 88,0 & 302,1 & $>$ & 115,9 & {$[\mathrm{M}+\mathrm{H}]^{+}$} & 20 & 18 & 12 & 15,99 \\
\hline 65 & Fenpiroximato & 422,3 & $>$ & 366,1 & 422,3 & $>$ & 134,9 & {$[\mathrm{M}+\mathrm{H}]^{+}$} & 30 & 17 & 31 & 19,17 \\
\hline 66 & Fenpropimorfe & 304,2 & $>$ & 147,0 & 304,2 & $>$ & 130,0 & {$[\mathrm{M}+\mathrm{H}]^{+}$} & 45 & 25 & 31 & 21,71 \\
\hline 67 & Fentiona & 279,0 & $>$ & 168,9 & 279,0 & $>$ & 104,9 & {$[\mathrm{M}+\mathrm{H}]^{+}$} & 25 & 25 & 18 & 16,54 \\
\hline 68 & Fentiona Sulfóxido & 294,7 & $>$ & 108,9 & 294,7 & $>$ & 279,7 & {$[\mathrm{M}+\mathrm{H}]^{+}$} & 30 & 30 & 15 & 12,11 \\
\hline 69 & Fluazifope-P-Butílico (s) & 384,0 & $>$ & 282,0 & 384,0 & $>$ & 328,0 & {$[\mathrm{M}+\mathrm{H}]^{+}$} & 25 & 25 & 20 & 17,51 \\
\hline 70 & Flufenacete & 364,1 & $>$ & 193,9 & 364,1 & $>$ & 151,9 & {$[\mathrm{M}+\mathrm{H}]^{+}$} & 20 & 18 & 10 & 15,47 \\
\hline 71 & Fluquinconazol (s) & 375,7 & $>$ & 348,8 & 375,7 & $>$ & 107,9 & {$[\mathrm{M}+\mathrm{H}]^{+}$} & 35 & 40 & 20 & 15,55 \\
\hline 72 & Flusilazole & 316,1 & $>$ & 247,0 & 316,1 & $>$ & 165,0 & {$[\mathrm{M}+\mathrm{H}]^{+}$} & 35 & 25 & 18 & 15,91 \\
\hline 73 & Flutriafol (s) & 302,1 & $>$ & 70,1 & 302,1 & $>$ & 122,9 & {$[\mathrm{M}+\mathrm{H}]^{+}$} & 25 & 16 & 30 & 13,10 \\
\hline 74 & Fosalona & 368,0 & $>$ & 182,0 & 368,0 & $>$ & 111,0 & {$[\mathrm{M}+\mathrm{H}]^{+}$} & 25 & 45 & 15 & 16,84 \\
\hline 75 & Fosmete & 317,9 & $>$ & 159,9 & 317,9 & $>$ & 132,9 & {$[\mathrm{M}+\mathrm{H}]^{+}$} & 20 & 40 & 20 & 14,25 \\
\hline 76 & Fostiazato & 284,1 & $>$ & 103,9 & 284,1 & $>$ & 227,8 & {$[\mathrm{M}+\mathrm{H}]^{+}$} & 20 & 22 & 10 & 12,79 \\
\hline 77 & Furatiocarbe & 383,2 & $>$ & 194,9 & 383,2 & $>$ & 252,0 & {$[\mathrm{M}+\mathrm{H}]^{+}$} & 25 & 18 & 12 & 17,69 \\
\hline 78 & Hexitiazoxi & 352,8 & $>$ & 227,8 & 352,8 & $>$ & 167,8 & {$[\mathrm{M}+\mathrm{H}]^{+}$} & 20 & 25 & 15 & 18,43 \\
\hline 79 & Imazalil & 297,1 & $>$ & 69,1 & 297,1 & $>$ & 158,9 & {$[\mathrm{M}+\mathrm{H}]^{+}$} & 35 & 18 & 24 & 16,75 \\
\hline 80 & Imidacloprido (s) & 256,1 & $>$ & 174,9 & 256,1 & $>$ & 209,0 & {$[\mathrm{M}+\mathrm{H}]^{+}$} & 25 & 20 & 12 & 7,65 \\
\hline 81 & Indoxacarbe & 528,1 & $>$ & 202,9 & 528,1 & $>$ & 217,9 & {$[\mathrm{M}+\mathrm{H}]^{+}$} & 30 & 40 & 25 & 16,75 \\
\hline 82 & Iprovalicarbe & 321,2 & $>$ & 118,9 & 321,2 & $>$ & 203,0 & {$[\mathrm{M}+\mathrm{H}]^{+}$} & 20 & 18 & 10 & 15,38 \\
\hline 83 & Isoprotiolane & 291,1 & $>$ & 188,8 & 291,1 & $>$ & 230,9 & {$[\mathrm{M}+\mathrm{H}]^{+}$} & 20 & 22 & 12 & 15,08 \\
\hline 84 & Isoxaflutol & 359,5 & $>$ & 250,6 & 359,5 & $>$ & 220,0 & {$[\mathrm{M}+\mathrm{H}]^{+}$} & 20 & 35 & 15 & 13,34 \\
\hline 85 & Isoxationa & 313,7 & $>$ & 104,7 & 313,7 & $>$ & 285,5 & {$[\mathrm{M}+\mathrm{H}]^{+}$} & 20 & 15 & 10 & 16,83 \\
\hline 86 & Linuron (s) & 249,0 & $>$ & 159,9 & 249,0 & $>$ & 181,9 & {$[\mathrm{M}+\mathrm{H}]^{+}$} & 20 & 20 & 16 & 14,87 \\
\hline 87 & Malationa & 331,0 & $>$ & 126,9 & 331,0 & $>$ & 98,9 & {$[\mathrm{M}+\mathrm{H}]^{+}$} & 20 & 25 & 12 & 14,93 \\
\hline 88 & Mefenacete & 299,1 & $>$ & 147,9 & 299,1 & $>$ & 119,9 & {$[\mathrm{M}+\mathrm{H}]^{+}$} & 20 & 25 & 16 & 15,38 \\
\hline 89 & Mefosfolan & 270,0 & $>$ & 139,8 & 270,0 & $>$ & 195,9 & {$[\mathrm{M}+\mathrm{H}]^{+}$} & 35 & 25 & 14 & 11,41 \\
\hline 90 & Mepronil & 270,1 & $>$ & 118,9 & 270,1 & $>$ & 91,0 & {$[\mathrm{M}+\mathrm{H}]^{+}$} & 30 & 40 & 25 & 15,08 \\
\hline 91 & Metalaxil & 280,1 & $>$ & 220,0 & 280,1 & $>$ & 192,0 & {$[\mathrm{M}+\mathrm{H}]^{+}$} & 25 & 18 & 12 & 13,44 \\
\hline 92 & Metamidofós (s) & 141,9 & $>$ & 93,9 & 141,9 & $>$ & 124,8 & {$[\mathrm{M}+\mathrm{H}]^{+}$} & 30 & 12 & 14 & 3,94 \\
\hline 93 & Metconazol (s) & 320,1 & $>$ & 70,1 & 320,1 & $>$ & 124,8 & {$[\mathrm{M}+\mathrm{H}]^{+}$} & 35 & 18 & 40 & 16,83 \\
\hline
\end{tabular}


Gouvêa AV, Cardoso MHWM, Bastos LHP, Silva CB, Ortiz ND, Nóbrega AWE, et al. Validação de metodologia QuEChERS-acetato para a análise de multirresíduo de agrotóxicos em amostras de soja e de extrato solúvel de soja utilizando cromatografia líquida de alta eficiência acoplada à espectrometria de massas sequencial. Rev Inst Adolfo Lutz. São Paulo, 2014; 73(1):40-58.

\begin{tabular}{|c|c|c|c|c|c|c|c|c|c|c|c|c|}
\hline \multicolumn{13}{|c|}{ Cont. Tabela 1} \\
\hline \multirow{2}{*}{$\begin{array}{l}\text { No }^{\circ} \\
94\end{array}$} & \multirow{2}{*}{$\begin{array}{l}\text { Substância } \\
\text { Metidationa }\end{array}$} & \multicolumn{2}{|c|}{$\begin{array}{c}\text { Transição } \\
\text { Quantificação } \\
\text { MRM }\end{array}$} & \multicolumn{2}{|c|}{$\begin{array}{c}\text { Transição } \\
\text { Confirmação } \\
\text { MRM }\end{array}$} & \multicolumn{2}{|c|}{$\begin{array}{l}\text { Espécie } \\
\text { do Íon } \\
\text { Precursor }\end{array}$} & \multirow{2}{*}{$\begin{array}{c}\begin{array}{c}\text { Espécie } \\
\text { do Íon } \\
\text { Precursor }\end{array} \\
{[\mathrm{M}+\mathrm{H}]^{+}}\end{array}$} & \multirow{2}{*}{$\begin{array}{c}\begin{array}{c}\text { Voltagem } \\
\text { do Cone } \\
(\mathrm{V})\end{array} \\
20\end{array}$} & \multirow{2}{*}{$\begin{array}{c}\begin{array}{c}\text { Energia de } \\
\text { Colisão } \\
\text { Quant. } \\
(\mathrm{eV})\end{array} \\
22\end{array}$} & \multirow{2}{*}{$\begin{array}{c}\begin{array}{c}\text { Energia de } \\
\text { Colisão } \\
\text { Confirm. } \\
(\mathrm{eV})\end{array} \\
12\end{array}$} & \multirow{2}{*}{$\begin{array}{c}\begin{array}{c}\mathbf{T r} \\
(\mathbf{m i n})\end{array} \\
14,05\end{array}$} \\
\hline & & 303,0 & $>$ & 84,9 & 303,0 & $>$ & 144,8 & & & & & \\
\hline 95 & Metiocarbe & 226,1 & $>$ & 121,0 & 226,1 & $>$ & 168,9 & {$[\mathrm{M}+\mathrm{H}]^{+}$} & 20 & 20 & 10 & 14,82 \\
\hline 96 & Metiocarbe Sulfona & 275,1 & $>$ & 121,9 & 275,1 & $>$ & 200,9 & {$[\mathrm{M}+\mathrm{NH} 4]^{+}$} & 15 & 24 & 14 & 8,84 \\
\hline 97 & Metiocarbe Sulfóxido & 242,1 & $>$ & 184,9 & 242,1 & $>$ & 121,9 & {$[\mathrm{M}+\mathrm{H}]^{+}$} & 25 & 30 & 14 & 7,91 \\
\hline 98 & Metobromuron & 258,5 & $>$ & 147,7 & 258,5 & $>$ & 169,6 & {$[\mathrm{M}+\mathrm{H}]^{+}$} & 20 & 15 & 20 & 13,44 \\
\hline 99 & Metomil (s) & 162,9 & $>$ & 105,9 & 162,9 & $>$ & 88,0 & {$[\mathrm{M}+\mathrm{H}]^{+}$} & 15 & 10 & 10 & 6,25 \\
\hline 100 & Metoxifenozida (s) & 368,8 & $>$ & 148,7 & 368,8 & $>$ & 312,8 & {$[\mathrm{M}+\mathrm{H}]^{+}$} & 15 & 15 & 10 & 14,93 \\
\hline 101 & Mevinfós & 225,0 & $>$ & 193,0 & 225,0 & $>$ & 127,0 & {$[\mathrm{M}+\mathrm{H}]^{+}$} & 20 & 15 & 10 & 8,42 \\
\hline 102 & Miclobutanil (s) & 289,1 & $>$ & 70,1 & 289,1 & $>$ & 124,9 & {$[\mathrm{M}+\mathrm{H}]^{+}$} & 30 & 18 & 30 & 15,08 \\
\hline 103 & Monocrotofos & 224,0 & $>$ & 126,8 & 224,0 & $>$ & 97,9 & {$[\mathrm{M}+\mathrm{H}]^{+}$} & 20 & 14 & 16 & 6,52 \\
\hline 104 & Ometoato & 214,0 & $>$ & 124,8 & 214,0 & $>$ & 182,8 & {$[\mathrm{M}+\mathrm{H}]^{+}$} & 25 & 22 & 10 & 4,52 \\
\hline 105 & Oxadixil & 279,1 & $>$ & 219,0 & 279,1 & $>$ & 132,3 & {$[\mathrm{M}+\mathrm{H}]^{+}$} & 20 & 25 & 12 & 10,51 \\
\hline 106 & Oxamil-Oxima & 162,9 & $>$ & 72,0 & 162,9 & $>$ & 89,9 & {$[\mathrm{M}+\mathrm{H}]^{+}$} & 20 & 12 & 16 & 4,62 \\
\hline 107 & Paclobutrazol & 294,1 & $>$ & 70,0 & 294,1 & $>$ & 124,9 & {$[\mathrm{M}+\mathrm{H}]^{+}$} & 30 & 18 & 35 & 14,92 \\
\hline 108 & Pencicurom & 329,1 & $>$ & 124,9 & 329,1 & $>$ & 218,0 & {$[\mathrm{M}+\mathrm{H}]^{+}$} & 30 & 30 & 16 & 17,01 \\
\hline 109 & Picoxistrobina (s) & 368,1 & $>$ & 144,9 & 368,1 & $>$ & 204,9 & {$[\mathrm{M}+\mathrm{H}]^{+}$} & 15 & 25 & 10 & 15,88 \\
\hline 110 & Pimetrozina & 218,1 & $>$ & 104,9 & 218,1 & $>$ & 78,3 & {$\left[\mathrm{M}+\mathrm{H}^{]+}\right.$} & 30 & 35 & 18 & 6,07 \\
\hline 111 & Butóxido de Piperonila & 356,3 & $>$ & 176,9 & 356,3 & $>$ & 119,0 & {$[\mathrm{M}+\mathrm{NH} 4]^{+}$} & 20 & 37 & 11 & 18,20 \\
\hline 112 & Piraclostrobina (s) & 388,1 & $>$ & 163,0 & 388,1 & $>$ & 193,9 & {$[\mathrm{M}+\mathrm{H}]^{+}$} & 25 & 25 & 12 & 16,75 \\
\hline 113 & Pirazofós & 374,0 & $>$ & 222,0 & 374,0 & $>$ & 194,0 & {$[\mathrm{M}+\mathrm{H}]^{+}$} & 40 & 35 & 20 & 17,08 \\
\hline 114 & Piridabem & 365,2 & $>$ & 147,0 & 365,2 & $>$ & 309,0 & {$[\mathrm{M}+\mathrm{H}]^{+}$} & 25 & 27 & 13 & 19,59 \\
\hline 115 & Piridafentiona & 341,1 & $>$ & 188,9 & 341,1 & $>$ & 92,0 & {$[\mathrm{M}+\mathrm{H}]^{+}$} & 35 & 40 & 25 & 15,18 \\
\hline 116 & Pirifenoxi & 295,1 & $>$ & 92,9 & 295,1 & $>$ & 66,1 & {$[\mathrm{M}+\mathrm{H}]^{+}$} & 35 & 40 & 22 & 16,07 \\
\hline 117 & Pirimetanil & 200,1 & $>$ & 106,9 & 200,1 & $>$ & 82,0 & {$[\mathrm{M}+\mathrm{H}]^{+}$} & 45 & 25 & 25 & 15,38 \\
\hline 118 & Pirimicarbe & 239,1 & $>$ & 72,0 & 239,1 & $>$ & 182,0 & {$[\mathrm{M}+\mathrm{H}]^{+}$} & 30 & 20 & 16 & 13,20 \\
\hline 119 & Pirimifós-Etílico & 333,5 & $>$ & 197,7 & 333,5 & $>$ & 305,5 & {$[\mathrm{M}+\mathrm{H}]^{+}$} & 35 & 25 & 18 & 18,11 \\
\hline 120 & Pirimifós-Metílico & 306,1 & $>$ & 107,9 & 306,1 & $>$ & 67,1 & {$[\mathrm{M}+\mathrm{H}]^{+}$} & 30 & 40 & 30 & 17,08 \\
\hline 121 & Piriproxifem (s) & 322,2 & $>$ & 95,9 & 322,2 & $>$ & 184,9 & {$[\mathrm{M}+\mathrm{H}]^{+}$} & 25 & 15 & 23 & 18,38 \\
\hline 122 & Procloraz & 376,0 & $>$ & 308,0 & 376,0 & $>$ & 266,0 & {$[\mathrm{M}+\mathrm{H}]^{+}$} & 20 & 15 & 15 & 17,01 \\
\hline 123 & Profenofós (s) & 374,8 & $>$ & 304,6 & 374,8 & $>$ & 346,7 & {$[\mathrm{M}+\mathrm{H}]^{+}$} & 25 & 20 & 15 & 17,82 \\
\hline 124 & Propargito & 368,3 & $>$ & 231,1 & 368,3 & $>$ & 175,0 & {$[\mathrm{M}+\mathrm{NH} 4]^{+}$} & 20 & 15 & 11 & 18,43 \\
\hline 125 & Propiconazol (s) & 342,1 & $>$ & 158,9 & 342,1 & $>$ & 69,1 & {$[\mathrm{M}+\mathrm{H}]^{+}$} & 35 & 20 & 30 & 16,66 \\
\hline 126 & Propizamida & 255,6 & $>$ & 189,6 & 255,6 & $>$ & 172,6 & {$[\mathrm{M}+\mathrm{H}]^{+}$} & 25 & 25 & 15 & 15,29 \\
\hline 127 & Propoxur & 210,1 & $>$ & 110,9 & 210,1 & $>$ & 92,9 & {$[\mathrm{M}+\mathrm{H}]^{+}$} & 15 & 25 & 12 & 11,73 \\
\hline 128 & Quinalfós & 298,8 & $>$ & 162,8 & 298,8 & $>$ & 146,8 & {$[\mathrm{M}+\mathrm{H}]^{+}$} & 25 & 20 & 25 & 16,56 \\
\hline 129 & Tebuconazol (s) & 307,8 & $>$ & 124,7 & 307,8 & $>$ & 69,9 & {$[\mathrm{M}+\mathrm{H}]^{+}$} & 30 & 20 & 35 & 16,37 \\
\hline 130 & Tebufenozide (s) & 352,8 & $>$ & 132,9 & 352,8 & $>$ & 296,8 & {$[\mathrm{M}+\mathrm{H}]^{+}$} & 10 & 20 & 10 & 15,88 \\
\hline 131 & Tebufenpirade & 334,2 & $>$ & 116,9 & 334,2 & $>$ & 144,9 & {$[\mathrm{M}+\mathrm{H}]^{+}$} & 45 & 35 & 25 & 17,88 \\
\hline 132 & Terbufós & 289,0 & $>$ & 103,0 & 289,0 & $>$ & 57,0 & {$[\mathrm{M}+\mathrm{H}]^{+}$} & 10 & 10 & 10 & 18,01 \\
\hline 133 & Tetraconazol (s) & 372,0 & $>$ & 158,9 & 372,0 & $>$ & 70,1 & {$[\mathrm{M}+\mathrm{H}]^{+}$} & 35 & 22 & 35 & 15,38 \\
\hline
\end{tabular}


Gouvêa AV, Cardoso MHWM, Bastos LHP, Silva CB, Ortiz ND, Nóbrega AWE, et al. Validação de metodologia QuEChERS-acetato para a análise de multirresíduo de agrotóxicos em amostras de soja e de extrato solúvel de soja utilizando cromatografia líquida de alta eficiência acoplada à espectrometria de massas sequencial. Rev Inst Adolfo Lutz. São Paulo, 2014; 73(1):40-58.

\begin{tabular}{|c|c|c|c|c|c|c|c|c|c|c|c|c|}
\hline \multicolumn{13}{|c|}{ Cont. Tabela 1} \\
\hline \multirow{2}{*}{$\begin{array}{l}\text { No } \\
134 \\
\end{array}$} & \multirow{2}{*}{$\begin{array}{l}\text { Substância } \\
\text { Tiabendazol (s) }\end{array}$} & \multicolumn{2}{|c|}{$\begin{array}{c}\text { Transição } \\
\text { Quantificação } \\
\text { MRM }\end{array}$} & \multicolumn{2}{|c|}{$\begin{array}{c}\text { Transição } \\
\text { Confirmação } \\
\text { MRM }\end{array}$} & \multicolumn{2}{|c|}{$\begin{array}{l}\text { Espécie } \\
\text { do Íon } \\
\text { Precursor }\end{array}$} & \multirow{2}{*}{$\begin{array}{c}\begin{array}{c}\text { Espécie } \\
\text { do Íon } \\
\text { Precursor }\end{array} \\
{[\mathrm{M}+\mathrm{H}]^{+}}\end{array}$} & \multirow{2}{*}{$\begin{array}{c}\begin{array}{c}\text { Voltagem } \\
\text { do Cone } \\
\text { (V) }\end{array} \\
45\end{array}$} & \multirow{2}{*}{$\begin{array}{c}\begin{array}{c}\text { Energia de } \\
\text { Colisão } \\
\text { Quant. } \\
(\mathrm{eV})\end{array} \\
30 \\
\end{array}$} & \multirow{2}{*}{$\begin{array}{c}\begin{array}{c}\text { Energia de } \\
\text { Colisão } \\
\text { Confirm. } \\
(\mathrm{eV})\end{array} \\
25 \\
\end{array}$} & \multirow{2}{*}{$\begin{array}{c}\begin{array}{c}\mathrm{Tr} \\
(\mathrm{min}\end{array} \\
12,15\end{array}$} \\
\hline & & 202,0 & $>$ & 174,9 & 202,0 & $>$ & 130,9 & & & & & \\
\hline 135 & Tiacloprido (s) & & $>$ & 125,8 & 253,0 & $>$ & 90,0 & {$[\mathrm{M}+\mathrm{H}]^{+}$} & 35 & 40 & 20 & 9,57 \\
\hline 136 & Tiametoxam (s) & 292,1 & $>$ & 210,9 & 292,1 & $>$ & 180,9 & {$[\mathrm{M}+\mathrm{H}]^{+}$} & 20 & 22 & 12 & 6,43 \\
\hline 137 & Tiodicarbe (s) & 355,0 & $>$ & 87,9 & 355,0 & $>$ & 107,9 & {$[\mathrm{M}+\mathrm{H}]^{+}$} & 20 & 16 & 16 & 12,59 \\
\hline 138 & Tiofanox-Sulfona & 268,1 & $>$ & 76,0 & 268,1 & $>$ & 57,2 & {$[\mathrm{M}+\mathrm{NH} 4]^{+}$} & 10 & 12 & 10 & 8,02 \\
\hline 139 & Triazofós (s) & 314,1 & $>$ & 161,9 & 314,1 & $>$ & 118,9 & {$[\mathrm{M}+\mathrm{H}]^{+}$} & 25 & 35 & 18 & 15,29 \\
\hline 140 & Triclorfom & 256,9 & $>$ & 108,8 & 256,9 & $>$ & 126,8 & {$[\mathrm{M}+\mathrm{H}]^{+}$} & 25 & 20 & 18 & 8,73 \\
\hline 141 & Trifloxistrobina (s) & 409,2 & $>$ & 185,9 & 409,2 & $>$ & 145,0 & {$[\mathrm{M}+\mathrm{H}]^{+}$} & 25 & 40 & 14 & 17,01 \\
\hline 142 & Triflumizol & 346,1 & $>$ & 278,0 & 346,1 & $>$ & 73,1 & {$[\mathrm{M}+\mathrm{H}]^{+}$} & 15 & 18 & 10 & 17,43 \\
\hline 143 & Vamidotiona & 287,7 & $>$ & 145,8 & 287,7 & $>$ & 117,5 & {$[\mathrm{M}+\mathrm{H}]^{+}$} & 20 & 25 & 12 & 8,20 \\
\hline 144 & Zoxamida & 336,0 & $>$ & 186,9 & 336,0 & $>$ & 158,9 & {$[\mathrm{M}+\mathrm{H}]^{+}$} & 30 & 40 & 25 & 16,75 \\
\hline
\end{tabular}

do trabalho foram: balanças analíticas modelos XP205 e AG245 (precisão de 5 casas decimais) fabricante Mettler Toledo (EUA), agitador modelo MS3 Digital e processador de sólidos modelo M20 fabricante IKA (EUA), banho ultrassom modelo 2510RMTM fabricante Branson Ultrasonics (EUA), centrífuga modelo T16 fabricante Beckman, liquidificador industrial modelo 36BL55 fabricante Ametek (EUA) e unidade de evaporação com fluxo de nitrogênio modelo Reacti Therm III Reacti Vap III fabricante Thermo Fisher Cientific (EUA).

Os gases utilizados para o estudo foram o nitrogênio e o argônio com pureza maior que 99,995 \% e $99,999 \%$, respectivamente.

\section{Método de extração QuEChERS-acetato}

O método multirresíduo QuEChERS-acetato foi utilizado para a validação nas matrizes soja e extrato solúvel de soja adquiridas em mercado local ${ }^{9,21}$.

Para a matriz soja foi feita a extração de $2 \mathrm{~g}$ de amostra com $3 \mathrm{~mL}$ de água adicionados à $15 \mathrm{~mL}$ de uma solução de acetonitrila com $1 \%$ de ácido acético glacial. Após agitação foi adicionado $6 \mathrm{~g}$ de sulfato de magnésio anidro e $1,5 \mathrm{~g}$ de acetato de sódio e novamente agitado seguido de uma centrifugação (3000 RPM, 5 min, $20^{\circ} \mathrm{C}$ ). Em uma alíquota de $2 \mathrm{~mL}$ do sobrenadante foi adicionado $100 \mathrm{mg}$ de sulfato de magnésio anidro e $50 \mathrm{mg}$ de PSA sendo promovidas uma nova agitação e centrifugação ( $3000 \mathrm{RPM}, 5 \mathrm{~min}, 20^{\circ} \mathrm{C}$ ). Após este procedimento $1 \mathrm{~mL}$ do sobrenadante foi evaporado sob atmosfera de nitrogênio e o resíduo foi reconstituído com metanol. Esta solução foi filtrada em filtro para solventes orgânicos com tamanho de partícula $0,22 \mu \mathrm{m}$ e injetado no cromatógrafo líquido com detector por massas sequencial.

Para a matriz extrato solúvel de soja o processo se inicia com a extração de $15 \mathrm{~g}$ de amostra direto com a solução de acetonitrila com $1 \%$ de ácido acético. $\mathrm{O}$ restante do procedimento segue a mesma sequência anteriormente descrita.

\section{Validação do método QuEChERS-acetato}

Os parâmetros avaliados no processo de validação do método foram: seletividade, linearidade, faixa de trabalho e faixa linear, exatidão (recuperação) e precisão (repetitividade), limite de detecção e de quantificação.

A seletividade foi verificada analisando-se uma amostra de soja e outra de extrato solúvel de soja. Ambas foram avaliadas quanto à presença de interferentes das substâncias selecionadas para o estudo. Foram preparadas soluções em metanol nas concentrações nominais anteriormente descritas para determinação da faixa de trabalho das curvas analíticas. Foram evaporados $1 \mathrm{~mL}$ do extrato branco da soja e do extrato solúvel de soja e adicionados aos resíduos da evaporação os pontos das curvas analíticas sendo assim feita a avaliação das curvas analíticas nas duas matrizes. Para determinação da faixa de trabalho linear das curvas analíticas, foram considerados valores adequados de $R^{2} \geq 0,95$ e $r \geq 0,98$, regressão linear significativa $(\mathrm{p}<0,001)$ e homogeneidade dos resíduos $(\alpha=0,05)$. O efeito matriz foi calculado considerando-se as curvas preparadas em metanol e nas matrizes soja e extrato solúvel de soja. Foi utilizada 
a planilha desenvolvida por Bazilio et $\mathrm{al}^{14}$ para todos os cálculos de linearidade das curvas analíticas.

Para a avaliação da exatidão (recuperação) e precisão (repetitividade) foram utilizados ensaios de fortificação com as amostras avaliadas anteriormente no parâmetro seletividade. A amostra de soja foi fortificada em dois níveis nas concentrações nominais de $0,01 \mathrm{e}$ $0,2 \mathrm{mg} \cdot \mathrm{kg}^{-1}$ com quatro replicatas de cada nível. Para a amostra de extrato solúvel de soja também foram fortificados dois níveis, porém com concentrações nominais de 0,01 e $0,03 \mathrm{mg} \cdot \mathrm{kg}^{-1} \mathrm{em}$ quatro replicatas de cada nível. Foram pesadas oito amostras de soja com duas gramas. Essas amostras foram separadas em dois grupos de quatro amostras. No primeiro grupo foram adicionados a cada uma das quatro amostras uma solução de fortificação de concentração nominal de $0,02 \mu \mathrm{g} \cdot \mathrm{mL}^{-1}$ correspondendo ao $1^{\circ}$ nível de fortificação com quatro replicatas na concentração nominal final de 0,01 mg. $\mathrm{kg}^{-1}$. Para obtenção desse nível de fortificação a alíquota de evaporação foi de $2 \mathrm{~mL}$. No segundo grupo de amostras foram adicionados a cada uma das quatro amostras uma solução de fortificação de $0,2 \mu \mathrm{g} \cdot \mathrm{mL}^{-1}$ correspondendo ao $2^{\circ}$ nível de fortificação com quatro replicatas na concentração nominal final de $0,2 \mathrm{mg} \cdot \mathrm{kg}^{-1}$. Para obtenção desse nível de fortificação o volume de acetonitrila adicionado foi de $10 \mathrm{~mL}$. Para o extrato solúvel de soja foram pesadas também oito amostras de soja com quinze gramas. Essas amostras foram separadas em dois grupos de quatro amostras. No primeiro grupo foram adicionados a cada uma das quatro amostras uma solução de fortificação de concentração nominal de $0,02 \mu \mathrm{g} \cdot \mathrm{mL}^{-1}$ correspondendo ao $1^{\circ}$ nível de fortificação com quatro replicatas na concentração nominal final de $0,01 \mathrm{mg} \cdot \mathrm{kg}^{-1}$. No segundo grupo de amostras foram adicionados a cada uma das quatro amostras uma solução de fortificação de $0,2 \mu \mathrm{g} \cdot \mathrm{mL}^{-1}$ correspondendo ao $2^{\circ}$ nível de fortificação com quatro replicatas na concentração nominal final de $0,03 \mathrm{mg} \cdot \mathrm{kg}^{-1}$. Os critérios de aceitação para esses parâmetros de desempenho, definidos pelo $\mathrm{SANCO}^{18}$ são limites de 70 a $120 \%$ de recuperação e valores aceitáveis de $\operatorname{DPR}(\%)$ ou $\mathrm{CV}(\%) \leq 20 \%$. O limite de quantificação foi definido como sendo a menor concentração adicionada na matriz que obteve resultado satisfatório para os parâmetros de linearidade, exatidão, precisão e uma relação $S / R$ maior que 10.

\section{RESULTADOS E DISCUSSÃO}

\section{Avaliação das curvas analíticas}

Das cento e quarenta e quatro substâncias estudadas, quatro delas (benalaxil, dodemorfe, espinosade e quinalfós) apresentaram resultados insatisfatórios de acordo com a avaliação dos critérios estabelecidos nas condições analíticas do trabalho. O mesmo comportamento das substâncias nas curvas preparadas em solvente e nas preparadas na matriz soja foi observado. Para a curva preparada na matriz extrato solúvel de soja três substâncias (dissulfotom, fentiona e pirifenoxi) apresentaram resultados insatisfatórios além das quatro já observadas no estudo da matriz soja.

As substâncias com resultados satisfatórios para todos os critérios estabelecidos representaram 97 \% (140) da totalidade para a soja e $95 \%$ (137) para o extrato solúvel de soja.

As curvas analíticas estudadas demonstraram ser adequadas para o estudo de acordo com os critérios estabelecidos. Além da quantificação com curvas o documento do $\mathrm{SANCO}^{18}$ também permite a quantificação através de um cálculo pontual, ou seja, com apenas um ponto da curva, os critérios estabelecidos pelo documento são: o ponto pode ter uma concentração com uma variação permitida de $\pm 20 \%$ em relação à concentração da substância na amostra se o LMR é excedido, quando o LMR não é excedido essa variação pode ser de até $\pm 50 \%$.

Além disso, a quantificação pontual pode fornecer resultados mais fidedignos do que a quantificação com a curva analítica se a resposta do detector é variável com o tempo ${ }^{18}$.

Quanto ao efeito matriz, foi observado que em trinta e cinco (24\%) das substâncias possuíam tal interferência para a matriz soja e cinco (3\%) para a matriz extrato solúvel de soja.

\section{Avaliação da exatidão, precisão e definição do limite de detecção e quantificação}

$\mathrm{Na}$ matriz soja, das cento e quarenta e quatro substâncias estudadas, dezenove agrotóxicos (abamectina, carbossulfano, ciproconazol, ciromazina, clorfenvinfós, clorpirifós, diclorvós, dissulfotom, diurom, espinosade, fenazaquina, fentiona, fosmete, isoxaflutol, linuron, metamidofós, metomil, pirifenoxi e tiobencarbe) representando $13 \%$ do total das substâncias selecionadas para o estudo obtiveram resultados insatisfatórios de acordo com os critérios estabelecidos para os ensaios 
de exatidão e de precisão, dentre eles o ciproconazol e o espinosade que são permitidos para o uso na cultura. Para o extrato solúvel de soja quinze substâncias (abamectina, diclorvós, diuron, epoxiconazol, espinosade, etirimol, famoxadona, fenpiroximato, fentiona, fosmete, imazalil, isoprotiolana, isoxaflutol, pirimifós-etílico e terbufós) representando $10 \%$ do total das substâncias selecionadas para o estudo tiveram o mesmo comportamento sendo que quatro delas (abamectina, diurom, epoxiconasol e espinosade) são permitidas para o uso na cultura da soja. Do total das cento e quarenta e quatro substâncias estudadas, setenta e cinco substâncias (52\% do total) tiveram resultados satisfatórios para ambas as matrizes estudadas. Cinco (3,5\% do total) apenas para a matriz soja e quarenta e quatro ( $31 \%$ do total) apenas para a matriz extrato solúvel de soja. Para a matriz soja oitenta substâncias (55 \% do total) tiveram resultados satisfatórios enquanto que para a matriz extrato solúvel de soja o total foi de cento e dezenove substâncias ( $83 \%$ do total). Estes resultados estão descritos nas Tabelas $2 \mathrm{e}$ 3. A letra "s" ao lado do nome das substâncias indica o uso permitido para a cultura da soja.

Para as substâncias presentes nas Tabelas $4 \mathrm{e}$ 5 a avaliação de exatidão e de precisão foi realizada

Tabela 2. Resultados da avaliação da exatidão, precisão e limite de detecção e quantificação para a matriz soja

\begin{tabular}{|c|c|c|c|c|c|}
\hline \multirow[t]{2}{*}{ Substância } & \multicolumn{2}{|c|}{$\begin{array}{c}\text { Exatidão - } \\
\text { Recuperação (\%) }\end{array}$} & \multicolumn{2}{|c|}{$\begin{array}{c}\text { Precisão - } \\
\text { Repetitividade } \\
\text { CV (\%) }\end{array}$} & \multirow{2}{*}{$\begin{array}{c}\text { Limite de } \\
\text { Quantificação } \\
\text { mg.kg-1 }\end{array}$} \\
\hline & $1^{\circ}$ Nível & $2^{\circ}$ Nível & $1^{\circ}$ Nível & $2^{\circ}$ Nível & \\
\hline Aldicarbe & 90 & 89 & 17 & 4 & 0,010 \\
\hline Aldicarbe Sulfona & 80 & 89 & 12 & 4 & 0,010 \\
\hline Aldicarbe Sulfóxido & 79 & 87 & 3 & 4 & 0,010 \\
\hline Ametrina & 90 & 83 & 9 & 3 & 0,010 \\
\hline Azaconazole & 88 & 83 & 3 & 7 & 0,010 \\
\hline Azinfós-Metílico & 79 & 92 & 8 & 9 & 0,010 \\
\hline Azoxistrobina (s) & 90 & 95 & 1 & 6 & 0,010 \\
\hline Boscalida & 77 & 83 & 12 & 12 & 0,010 \\
\hline Buprofenzina (s) & 80 & 88 & 4 & 8 & 0,010 \\
\hline Butocarboxim Sulfóxido & 83 & 89 & 3 & 4 & 0,010 \\
\hline Cadusafós & 88 & 94 & 15 & 3 & 0,010 \\
\hline Carbaril & 87 & 95 & 5 & 3 & 0,010 \\
\hline Carbendazim (s) & 76 & 89 & 5 & 4 & 0,010 \\
\hline Carbofurano & 84 & 89 & 1 & 3 & 0,010 \\
\hline Ciazofamida & 86 & 102 & 14 & 5 & 0,011 \\
\hline Cimoxanil & 78 & 82 & 8 & 7 & 0,010 \\
\hline Ciprodinil & 101 & 90 & 1 & 1 & 0,010 \\
\hline Clotianidina (s) & 83 & 108 & 2 & 5 & 0,010 \\
\hline Diazinona & 118 & 92 & 5 & 2 & 0,014 \\
\hline Dimetomorfe & 103 & 81 & 3 & 4 & 0,010 \\
\hline Diniconazol & 89 & 79 & 3 & 9 & 0,012 \\
\hline Etiofencarbe-Sulfona & 78 & 94 & 9 & 5 & 0,010 \\
\hline Etiofencarbe-Sulfoxido & 77 & 90 & 3 & 4 & 0,008 \\
\hline Etiona & 82 & 82 & 4 & 10 & 0,010 \\
\hline Etiprole & 77 & 85 & 8 & 7 & 0,010 \\
\hline Fenamidona & 77 & 94 & 5 & 4 & 0,010 \\
\hline Fenhexamide & 86 & 70 & 8 & 8 & 0,010 \\
\hline Fenpiroximato & 70 & 70 & 3 & 12 & 0,010 \\
\hline
\end{tabular}


Gouvêa AV, Cardoso MHWM, Bastos LHP, Silva CB, Ortiz ND, Nóbrega AWE, et al. Validação de metodologia QuEChERS-acetato para a análise de multirresíduo de agrotóxicos em amostras de soja e de extrato solúvel de soja utilizando cromatografia líquida de alta eficiência acoplada à espectrometria de massas sequencial.

Rev Inst Adolfo Lutz. São Paulo, 2014; 73(1):40-58.

\begin{tabular}{|c|c|c|c|c|c|}
\hline \multicolumn{6}{|l|}{ Cont. Tabela 2} \\
\hline \multirow[t]{2}{*}{ Substância } & \multicolumn{2}{|c|}{$\begin{array}{c}\text { Exatidão - } \\
\text { Recuperação (\%) }\end{array}$} & \multicolumn{2}{|c|}{$\begin{array}{c}\text { Precisão - } \\
\text { Repetitividade } \\
\text { CV (\%) }\end{array}$} & \multirow{2}{*}{$\begin{array}{c}\text { Limite de } \\
\text { Quantificação } \\
\text { mg.kg-1 }\end{array}$} \\
\hline & $1^{\circ}$ Nível & $2^{\circ}$ Nível & $1^{\circ}$ Nível & $2^{\circ}$ Nível & \\
\hline Fenpropimorfe & 81 & 83 & 1 & 5 & 0,010 \\
\hline Fentiona Sulfóxido & 83 & 89 & 6 & 5 & 0,010 \\
\hline Flufenacete & 85 & 90 & 1 & 7 & 0,010 \\
\hline Fluquinconazol (s) & 75 & 89 & 10 & 10 & 0,010 \\
\hline Flusilazole & 89 & 87 & 5 & 3 & 0,011 \\
\hline Flutriafol (s) & 73 & 92 & 5 & 4 & 0,010 \\
\hline Fosalona & 106 & 100 & 3 & 8 & 0,010 \\
\hline Fostiazato & 83 & 93 & 3 & 3 & 0,010 \\
\hline Furatiocarbe & 110 & 86 & 5 & 6 & 0,010 \\
\hline Hexitiazoxi & 86 & 70 & 5 & 7 & 0,010 \\
\hline Imazalil & 76 & 70 & 16 & 9 & 0,010 \\
\hline Imidacloprido (s) & 86 & 93 & 6 & 3 & 0,010 \\
\hline Iprovalicarbe & 88 & 113 & 7 & 9 & 0,010 \\
\hline Isoprotiolane & 80 & 97 & 10 & 7 & 0,010 \\
\hline Isoxationa & 91 & 88 & 5 & 8 & 0,010 \\
\hline Malationa & 106 & 92 & 4 & 8 & 0,010 \\
\hline Mefenacete & 90 & 96 & 2 & 7 & 0,010 \\
\hline Mefosfolan & 89 & 93 & 3 & 4 & 0,011 \\
\hline Mepronil & 118 & 95 & 3 & 4 & 0,010 \\
\hline Metalaxil & 86 & 102 & 1 & 4 & 0,010 \\
\hline Metconazol (s) & 83 & 90 & 6 & 4 & 0,010 \\
\hline Metiocarbe & 78 & 87 & 5 & 5 & 0,010 \\
\hline Metobromuron & 82 & 95 & 8 & 3 & 0,010 \\
\hline Ometoato & 78 & 84 & 4 & 3 & 0,010 \\
\hline Oxadixil & 95 & 100 & 2 & 1 & 0,010 \\
\hline Oxamil-Oxima & 71 & 90 & 5 & 5 & 0,010 \\
\hline Pencicurom & 84 & 97 & 2 & 4 & 0,010 \\
\hline Butóxido de Piperonila & 82 & 73 & 3 & 9 & 0,010 \\
\hline Piraclostrobina (s) & 119 & 95 & 4 & 5 & 0,010 \\
\hline Pirazofós & 110 & 76 & 4 & 2 & 0,010 \\
\hline Piridabem & 83 & 75 & 1 & 7 & 0,010 \\
\hline Piridafentiona & 107 & 90 & 1 & 2 & 0,010 \\
\hline Pirimetanil & 89 & 91 & 13 & 4 & 0,005 \\
\hline Pirimicarbe & 87 & 96 & 3 & 3 & 0,010 \\
\hline Pirimifós-Etílico & 85 & 81 & 10 & 10 & 0,010 \\
\hline Pirimifós-Metílico & 99 & 98 & 2 & 6 & 0,010 \\
\hline Piriproxifem (s) & 73 & 76 & 4 & 12 & 0,010 \\
\hline Procloraz & 90 & 86 & 3 & 9 & 0,010 \\
\hline Profenofós (s) & 77 & 71 & 5 & 6 & 0,010 \\
\hline Propargito & 80 & 83 & 5 & 8 & 0,009 \\
\hline Propiconazol (s) & 97 & 88 & 3 & 7 & 0,010 \\
\hline
\end{tabular}


Gouvêa AV, Cardoso MHWM, Bastos LHP, Silva CB, Ortiz ND, Nóbrega AWE, et al. Validação de metodologia QuEChERS-acetato para a análise de multirresíduo de agrotóxicos em amostras de soja e de extrato solúvel de soja utilizando cromatografia líquida de alta eficiência acoplada à espectrometria de massas sequencial. Rev Inst Adolfo Lutz. São Paulo, 2014; 73(1):40-58.

\begin{tabular}{|c|c|c|c|c|c|}
\hline \multicolumn{6}{|l|}{ Cont. Tabela 2} \\
\hline \multirow[t]{2}{*}{ Substância } & \multicolumn{2}{|c|}{$\begin{array}{c}\text { Exatidão - } \\
\text { Recuperação (\%) }\end{array}$} & \multicolumn{2}{|c|}{$\begin{array}{c}\text { Precisão - } \\
\text { Repetitividade } \\
\text { CV (\%) }\end{array}$} & \multirow{2}{*}{$\begin{array}{c}\text { Limite de } \\
\text { Quantificação } \\
\text { mg.kg-1 }\end{array}$} \\
\hline & $1^{\circ}$ Nível & $2^{\circ}$ Nível & $1^{\circ}$ Nível & $2^{\circ}$ Nível & \\
\hline Propoxur & 88 & 85 & 3 & 9 & 0,011 \\
\hline Tebufenpirade & 74 & 75 & 4 & 13 & 0,010 \\
\hline Terbufós & 87 & 93 & 4 & 13 & 0,010 \\
\hline Tetraconazol (s) & 86 & 91 & 5 & 8 & 0,005 \\
\hline Tiabendazol (s) & 74 & 75 & 9 & 5 & 0,010 \\
\hline Tiacloprido (s) & 93 & 94 & 7 & 7 & 0,010 \\
\hline Tiofanox-Sulfona & 81 & 98 & 3 & 4 & 0,010 \\
\hline Triazofós (s) & 114 & 90 & 3 & 6 & 0,010 \\
\hline Trifloxistrobina (s) & 107 & 92 & 2 & 4 & 0,010 \\
\hline Triflumizol & 93 & 88 & 2 & 9 & 0,011 \\
\hline Vamidotiona & 81 & 94 & 6 & 3 & 0,020 \\
\hline
\end{tabular}

Tabela 3. Resultados da avaliação da exatidão, precisão e limite de detecção e quantificação para a matriz extrato solúvel de soja

\begin{tabular}{|c|c|c|c|c|c|}
\hline \multirow[t]{2}{*}{ Substância } & \multicolumn{2}{|c|}{$\begin{array}{c}\text { Exatidão - } \\
\text { Recuperação (\%) }\end{array}$} & \multicolumn{2}{|c|}{$\begin{array}{c}\text { Precisão } \\
\text { Repetividade CV (\%) }\end{array}$} & \multirow{2}{*}{$\begin{array}{c}\text { Limite de } \\
\text { Quantificação } \\
\text { mg.kg-1 }\end{array}$} \\
\hline & $1^{\circ}$ Nível & $2^{\circ}$ Nível & $1^{\circ}$ Nível & $2^{\circ}$ Nível & \\
\hline 3-Hidroxi-Carbofurano & 96 & 85 & 3 & 6 & 0,012 \\
\hline Acefato (s) & 90 & 78 & 2 & 8 & 0,014 \\
\hline Acetamiprido (s) & 99 & 119 & 2 & 7 & 0,013 \\
\hline Aldicarbe & 102 & 88 & 2 & 10 & 0,013 \\
\hline Aldicarbe Sulfona & 99 & 82 & 1 & 5 & 0,013 \\
\hline Aldicarbe Sulfóxido & 96 & 84 & 3 & 8 & 0,013 \\
\hline Ametrina & 92 & 92 & 10 & 7 & 0,013 \\
\hline Azaconazole & 97 & 101 & 2 & 12 & 0,013 \\
\hline Azametifós & 94 & 86 & 1 & 6 & 0,013 \\
\hline Azinfós-Etílico & 114 & 96 & 5 & 13 & 0,013 \\
\hline Azinfós-Metílico & 102 & 88 & 3 & 8 & 0,013 \\
\hline Azoxistrobina (s) & 102 & 91 & 1 & 6 & 0,013 \\
\hline Boscalida & 94 & 84 & 5 & 11 & 0,013 \\
\hline Bromuconazol (s) & 97 & 87 & 6 & 15 & 0,013 \\
\hline Bupirimate & 111 & 101 & 4 & 10 & 0,013 \\
\hline Buprofenzina (s) & 94 & 82 & 2 & 5 & 0,013 \\
\hline Butocarboxim Sulfóxido & 91 & 80 & 4 & 7 & 0,013 \\
\hline Cadusafós & 88 & 78 & 4 & 8 & 0,013 \\
\hline Carbaril & 101 & 91 & 2 & 4 & 0,013 \\
\hline Carbendazim (s) & 95 & 81 & 2 & 6 & 0,013 \\
\hline Carbofurano & 100 & 88 & 2 & 6 & 0,013 \\
\hline Ciazofamida & 95 & 108 & 4 & 5 & 0,014 \\
\hline Cimoxanil & 101 & 89 & 7 & 7 & 0,013 \\
\hline Ciproconazol (s) & 112 & 93 & 6 & 12 & 0,013 \\
\hline
\end{tabular}


Gouvêa AV, Cardoso MHWM, Bastos LHP, Silva CB, Ortiz ND, Nóbrega AWE, et al. Validação de metodologia QuEChERS-acetato para a análise de multirresíduo de agrotóxicos em amostras de soja e de extrato solúvel de soja utilizando cromatografia líquida de alta eficiência acoplada à espectrometria de massas sequencial. Rev Inst Adolfo Lutz. São Paulo, 2014; 73(1):40-58.

\begin{tabular}{|c|c|c|c|c|c|}
\hline \multicolumn{6}{|l|}{ Cont. Tabela 3} \\
\hline \multirow[t]{2}{*}{ Substância } & \multicolumn{2}{|c|}{$\begin{array}{c}\text { Exatidão - } \\
\text { Recuperação (\%) }\end{array}$} & \multicolumn{2}{|c|}{$\begin{array}{c}\text { Precisão } \\
\text { Repetividade CV (\%) }\end{array}$} & \multirow{2}{*}{$\begin{array}{c}\text { Limite de } \\
\text { Quantificação } \\
\text { mg.kg-1 }\end{array}$} \\
\hline & $1{ }^{\circ}$ Nível & $2^{\circ}$ Nível & 10 Nível & $2^{\circ}$ Nível & \\
\hline Ciprodinil & 86 & 84 & 6 & 4 & 0,013 \\
\hline Ciromazina & 79 & 70 & 2 & 6 & 0,014 \\
\hline Clofentezina & 100 & 88 & 18 & 16 & 0,013 \\
\hline Clorbromuron & 103 & 84 & 7 & 12 & 0,013 \\
\hline Clorpirifós (s) & 88 & 76 & 9 & 12 & 0,013 \\
\hline Clotianidina (s) & 100 & 93 & 4 & 5 & 0,013 \\
\hline Coumafós & 84 & 84 & 5 & 11 & 0,013 \\
\hline Demeton-S-Metílico & 91 & 83 & 3 & 5 & 0,012 \\
\hline Desmedifam & 100 & 96 & 2 & 6 & 0,013 \\
\hline Diazinona & 89 & 75 & 2 & 8 & 0,018 \\
\hline Difenoconazol (s) & 102 & 84 & 2 & 3 & 0,013 \\
\hline Dimetoato & 92 & 112 & 1 & 7 & 0,013 \\
\hline Dimetomorfe & 104 & 81 & 4 & 8 & 0,013 \\
\hline Diniconazol & 96 & 83 & 8 & 4 & 0,015 \\
\hline Etiofencarbe-Sulfona & 102 & 87 & 2 & 7 & 0,013 \\
\hline Etiofencarbe-Sulfoxido & 96 & 84 & 2 & 9 & 0,010 \\
\hline Etiona & 99 & 81 & 2 & 4 & 0,013 \\
\hline Etiprole & 105 & 82 & 6 & 5 & 0,013 \\
\hline Etoprofós & 92 & 88 & 10 & 11 & 0,013 \\
\hline Etrinfós & 75 & 80 & 4 & 7 & 0,012 \\
\hline Fenamidona & 100 & 93 & 2 & 7 & 0,013 \\
\hline Fenamifós & 102 & 89 & 2 & 5 & 0,013 \\
\hline Fenazaquina & 91 & 92 & 4 & 7 & 0,013 \\
\hline Fenbuconazole & 109 & 116 & 2 & 8 & 0,013 \\
\hline Fenhexamide & 106 & 80 & 6 & 7 & 0,013 \\
\hline Fenoxicarbe & 102 & 91 & 6 & 6 & 0,013 \\
\hline Fenpropimorfe & 97 & 86 & 3 & 6 & 0,013 \\
\hline Fentiona Sulfóxido & 104 & 98 & 1 & 3 & 0,013 \\
\hline Fluazifope-P-Butílico (s) & 98 & 92 & 2 & 5 & 0,013 \\
\hline Flufenacete & 89 & 90 & 3 & 8 & 0,013 \\
\hline Fluquinconazol (s) & 95 & 71 & 15 & 9 & 0,013 \\
\hline Flusilazole & 116 & 94 & 7 & 8 & 0,014 \\
\hline Flutriafol (s) & 99 & 93 & 2 & 6 & 0,013 \\
\hline Fosalona & 103 & 99 & 4 & 7 & 0,013 \\
\hline Fostiazato & 100 & 89 & 3 & 5 & 0,013 \\
\hline Furatiocarbe & 97 & 84 & 4 & 5 & 0,013 \\
\hline Hexitiazoxi & 96 & 81 & 8 & 4 & 0,013 \\
\hline Imidacloprido (s) & 95 & 95 & 3 & 10 & 0,013 \\
\hline Indoxacarbe & 112 & 88 & 9 & 11 & 0,013 \\
\hline Iprovalicarbe & 103 & 91 & 2 & 5 & 0,013 \\
\hline
\end{tabular}


Gouvêa AV, Cardoso MHWM, Bastos LHP, Silva CB, Ortiz ND, Nóbrega AWE, et al. Validação de metodologia QuEChERS-acetato para a análise de multirresíduo de agrotóxicos em amostras de soja e de extrato solúvel de soja utilizando cromatografia líquida de alta eficiência acoplada à espectrometria de massas sequencial. Rev Inst Adolfo Lutz. São Paulo, 2014; 73(1):40-58.

\begin{tabular}{|c|c|c|c|c|c|}
\hline \multicolumn{6}{|l|}{ Cont. Tabela 3} \\
\hline \multirow[t]{2}{*}{ Substância } & \multicolumn{2}{|c|}{$\begin{array}{c}\text { Exatidão - } \\
\text { Recuperação (\%) }\end{array}$} & \multicolumn{2}{|c|}{$\begin{array}{c}\text { Precisão } \\
\text { Repetividade CV (\%) }\end{array}$} & \multirow{2}{*}{$\begin{array}{c}\text { Limite de } \\
\text { Quantificação } \\
\text { mg.kg-1 }\end{array}$} \\
\hline & $1^{\circ}$ Nível & $2^{\circ}$ Nível & $1^{\circ}$ Nível & $2^{\circ}$ Nível & \\
\hline Isoxationa & 92 & 80 & 10 & 4 & 0,014 \\
\hline Linuron (s) & 100 & 92 & 11 & 13 & 0,013 \\
\hline Malationa & 102 & 99 & 4 & 6 & 0,013 \\
\hline Mefenacete & 99 & 90 & 3 & 10 & 0,013 \\
\hline Mefosfolan & 100 & 85 & 2 & 6 & 0,014 \\
\hline Mepronil & 108 & 96 & 7 & 3 & 0,014 \\
\hline Metalaxil & 103 & 90 & 2 & 8 & 0,013 \\
\hline Metamidofós (s) & 70 & 70 & 3 & 5 & 0,015 \\
\hline Metconazol (s) & 103 & 78 & 3 & 2 & 0,013 \\
\hline Metidationa & 102 & 93 & 2 & 10 & 0,013 \\
\hline Metiocarbe & 103 & 89 & 2 & 5 & 0,013 \\
\hline Metiocarbe Sulfona & 95 & 93 & 2 & 6 & 0,010 \\
\hline Metiocarbe Sulfóxido & 97 & 82 & 3 & 7 & 0,013 \\
\hline Metobromuron & 106 & 100 & 2 & 5 & 0,013 \\
\hline Metomil (s) & 100 & 87 & 2 & 7 & 0,013 \\
\hline Metoxifenozida (s) & 104 & 97 & 2 & 6 & 0,013 \\
\hline Mevinfós & 81 & 82 & 6 & 7 & 0,013 \\
\hline Miclobutanil (s) & 88 & 109 & 3 & 6 & 0,013 \\
\hline Monocrotofos & 96 & 87 & 4 & 8 & 0,013 \\
\hline Ometoato & 95 & 81 & 2 & 7 & 0,013 \\
\hline Oxadixil & 99 & 86 & 2 & 6 & 0,013 \\
\hline Oxamil-Oxima & 95 & 90 & 2 & 8 & 0,013 \\
\hline Paclobutrazol & 108 & 85 & 1 & 7 & 0,015 \\
\hline Pencicurom & 97 & 87 & 2 & 4 & 0,013 \\
\hline Pimetrozina & 89 & 82 & 1 & 6 & 0,014 \\
\hline Butóxido de Piperonila & 98 & 82 & 2 & 2 & 0,014 \\
\hline Piraclostrobina (s) & 101 & 81 & 2 & 5 & 0,013 \\
\hline Pirazofós & 94 & 94 & 4 & 2 & 0,013 \\
\hline Piridabem & 90 & 82 & 6 & 5 & 0,013 \\
\hline Piridafentiona & 80 & 94 & 11 & 3 & 0,013 \\
\hline Pirimetanil & 97 & 84 & 1 & 1 & 0,006 \\
\hline Pirimicarbe & 97 & 92 & 3 & 6 & 0,013 \\
\hline Pirimifós-Metílico & 108 & 81 & 3 & 5 & 0,013 \\
\hline Piriproxifem (s) & 94 & 76 & 2 & 3 & 0,013 \\
\hline Procloraz & 107 & 82 & 11 & 8 & 0,013 \\
\hline Profenofós (s) & 97 & 78 & 5 & 5 & 0,013 \\
\hline Propargito & 96 & 81 & 2 & 10 & 0,012 \\
\hline Propiconazol (s) & 95 & 91 & 4 & 4 & 0,013 \\
\hline Propizamida & 114 & 87 & 9 & 12 & 0,013 \\
\hline Propoxur & 98 & 91 & 3 & 8 & 0,014 \\
\hline
\end{tabular}


Gouvêa AV, Cardoso MHWM, Bastos LHP, Silva CB, Ortiz ND, Nóbrega AWE, et al. Validação de metodologia QuEChERS-acetato para a análise de multirresíduo de agrotóxicos em amostras de soja e de extrato solúvel de soja utilizando cromatografia líquida de alta eficiência acoplada à espectrometria de massas sequencial. Rev Inst Adolfo Lutz. São Paulo, 2014; 73(1):40-58.

\begin{tabular}{|c|c|c|c|c|c|}
\hline \multirow{3}{*}{ Substância } & & & & & \\
\hline & \multicolumn{2}{|c|}{$\begin{array}{c}\text { Exatidão - } \\
\text { Recuperação (\%) }\end{array}$} & \multicolumn{2}{|c|}{$\begin{array}{c}\text { Precisão } \\
\text { Repetividade CV (\%) }\end{array}$} & \multirow{2}{*}{$\begin{array}{c}\text { Limite de } \\
\text { Quantificação } \\
\text { mg.kg-1 }\end{array}$} \\
\hline & 1 ' Nível & $2^{\circ}$ Nível & 10 Nível & $2^{\circ}$ Nível & \\
\hline Tebuconazol (s) & 93 & 97 & 13 & 9 & 0,013 \\
\hline Tebufenozide (s) & 104 & 94 & 8 & 5 & 0,013 \\
\hline Tebufenpirade & 99 & 76 & 3 & 5 & 0,014 \\
\hline Tetraconazol (s) & 96 & 94 & 2 & 2 & 0,006 \\
\hline Tiabendazol (s) & 99 & 90 & 1 & 5 & 0,013 \\
\hline Tiacloprido (s) & 100 & 89 & 2 & 7 & 0,013 \\
\hline Tiametoxam (s) & 102 & 93 & 3 & 8 & 0,007 \\
\hline Tiodicarbe (s) & 99 & 92 & 1 & 8 & 0,013 \\
\hline Tiofanox-Sulfona & 101 & 83 & 2 & 7 & 0,013 \\
\hline Triazofós (s) & 88 & 94 & 3 & 10 & 0,013 \\
\hline Triclorfom & 95 & 115 & 3 & 8 & 0,014 \\
\hline Trifloxistrobina (s) & 95 & 90 & 8 & 7 & 0,013 \\
\hline Triflumizol & 86 & 93 & 4 & 2 & 0,014 \\
\hline Vamidotiona & 97 & 87 & 2 & 7 & 0,026 \\
\hline Zoxamida & 112 & 84 & 7 & 7 & 0,012 \\
\hline
\end{tabular}

utilizando os valores encontrados no apenas no $2^{\circ}$ nível de fortificação destas substâncias. A letra "s" ao lado do nome das substâncias indica o uso permitido para a cultura da soja. Três substâncias $(2,1 \%$ do total $)$ apresentaram resultados satisfatórios no $2^{\circ}$ nível de fortificação para as duas matrizes estudadas. Para a matriz soja apenas trinta e nove substâncias ( $27 \%$ do total) apresentaram esse comportamento e duas $(1,4 \%$ do total) apenas para a matriz extrato solúvel de soja.

Tabela 4. Resultados da avaliação da exatidão, precisão e limite de detecção e quantificação avaliados no $2^{\circ}$ nível de fortificação para a matriz soja

\begin{tabular}{|c|c|c|c|}
\hline \multirow[t]{2}{*}{ Substância } & Exatidão - Recuperação (\%) & $\begin{array}{c}\text { Precisão - Repetitividade } \\
\text { CV (\%) }\end{array}$ & Limite de Quantificação \\
\hline & $2^{\circ}$ Nível & $2^{\circ}$ Nível & mg.kg- ${ }^{1}$ \\
\hline 3-Hidroxi-Carbofurano & 90 & 6 & 0,182 \\
\hline Acefato (s) & 77 & 3 & 0,199 \\
\hline Acetamiprido (s) & 92 & 7 & 0,188 \\
\hline Azametifós & 87 & 3 & 0,184 \\
\hline Azinfós-Etílico & 94 & 15 & 0,189 \\
\hline Bromuconazol (s) & 83 & 1 & 0,190 \\
\hline Bupirimate & 93 & 2 & 0,187 \\
\hline Clofentezina & 90 & 18 & 0,187 \\
\hline Clorbromuron & 103 & 11 & 0,187 \\
\hline Coumafós & 91 & 8 & 0,188 \\
\hline Cresoxim-Metílico (s) & 88 & 10 & 0,187 \\
\hline Demeton-S-Metílico & 87 & 4 & 0,180 \\
\hline Desmedifam & 92 & 3 & 0,187 \\
\hline Difenoconazol (s) & 85 & 5 & 0,190 \\
\hline
\end{tabular}


Gouvêa AV, Cardoso MHWM, Bastos LHP, Silva CB, Ortiz ND, Nóbrega AWE, et al. Validação de metodologia QuEChERS-acetato para a análise de multirresíduo de agrotóxicos em amostras de soja e de extrato solúvel de soja utilizando cromatografia líquida de alta eficiência acoplada à espectrometria de massas sequencial. Rev Inst Adolfo Lutz. São Paulo, 2014; 73(1):40-58.

\begin{tabular}{|c|c|c|c|}
\hline \multicolumn{4}{|l|}{ Cont. Tabela 4} \\
\hline \multirow[t]{2}{*}{ Substância } & Exatidão - Recuperação (\%) & $\begin{array}{c}\text { Precisão - Repetitividade } \\
\text { CV (\%) }\end{array}$ & Limite de Quantificação \\
\hline & $2^{\circ}$ Nível & $2^{\circ}$ Nível & mg.kg-1 ${ }^{1}$ \\
\hline Dimetoato & 98 & 3 & 0,187 \\
\hline Epoxiconazol (s) & 85 & 2 & 0,190 \\
\hline Etirimol & 78 & 11 & 0,189 \\
\hline Etoprofós & 83 & 8 & 0,190 \\
\hline Etrinfós & 82 & 9 & 0,178 \\
\hline Famoxadona & 88 & 2 & 0,187 \\
\hline Fenamifós & 99 & 5 & 0,188 \\
\hline Fenarimol (s) & 83 & 11 & 0,188 \\
\hline Fenbuconazole & 97 & 8 & 0,188 \\
\hline Fenoxicarbe & 90 & 3 & 0,190 \\
\hline Fluazifope-P-Butílico (s) & 90 & 4 & 0,187 \\
\hline Indoxacarbe & 99 & 14 & 0,187 \\
\hline Metidationa & 97 & 7 & 0,190 \\
\hline Metiocarbe Sulfona & 86 & 4 & 0,140 \\
\hline Metiocarbe Sulfóxido & 93 & 3 & 0,189 \\
\hline Metoxifenozida (s) & 90 & 3 & 0,187 \\
\hline Mevinfós & 90 & 9 & 0,192 \\
\hline Miclobutanil (s) & 104 & 4 & 0,189 \\
\hline Monocrotofos & 92 & 4 & 0,191 \\
\hline Paclobutrazol & 89 & 3 & 0,215 \\
\hline Picoxistrobina (s) & 93 & 8 & 0,204 \\
\hline Pimetrozina & 76 & 4 & 0,202 \\
\hline Propizamida & 91 & 11 & 0,191 \\
\hline Tebuconazol (s) & 85 & 6 & 0,194 \\
\hline Tebufenozide (s) & 99 & 12 & 0,187 \\
\hline Tiametoxam (s) & 96 & 5 & 0,104 \\
\hline Triclorfom & 89 & 6 & 0,203 \\
\hline Zoxamida & 95 & 5 & 0,182 \\
\hline
\end{tabular}

Tabela 5. Resultados da avaliação da exatidão, precisão e limite de detecção e quantificação avaliados no $2^{\circ}$ nível de fortificação para a matriz extrato solúvel de soja

\begin{tabular}{|c|c|c|c|}
\hline \multirow[t]{2}{*}{ Substância } & Exatidão - Recuperação (\%) & $\begin{array}{c}\text { Precisão - Repetitividade } \\
\text { CV (\%) }\end{array}$ & Limite de Quantificação \\
\hline & $2^{\circ}$ Nível & $2^{\circ}$ Nível & mg.kg- ${ }^{1}$ \\
\hline Carbossulfano (s) & 89 & 4 & 0,182 \\
\hline Clorfenvinfós & 82 & 14 & 0,027 \\
\hline Cresoxim-Metílico (s) & 77 & 8 & 0,026 \\
\hline Fenarimol (s) & 81 & 18 & 0,026 \\
\hline Picoxistrobina (s) & 90 & 7 & 0,028 \\
\hline
\end{tabular}


Os resultados das recuperações obtidas encontraram-se dentro dos limites de 70 a $120 \%$ definidos pelo $\mathrm{SANCO}^{18}$. E a precisão medida sob condições de repetitividade avaliada pelo coeficiente de variação $(\mathrm{CV}(\%))$ e/ou estimativa do desvio padrão relativo $(\mathrm{DPR}(\%))$ também encontraram-se dentro do limite de $20 \% 18$. O limite de quantificação foi adequado para as substâncias estudadas, pois seus valores definidos estão abaixo dos LMR destas substâncias permitidas para a cultura da soja e dentro do limite de sensibilidade do equipamento.

O método estudado, para cento e quarenta e quatro substâncias, foi considerado adequado, contemplando todos os critérios estabelecidos (estudo de linearidade, avaliação da precisão e exatidão) para cento e vinte e duas substâncias ( $85 \%$ do total) estudadas na matriz soja e cento e vinte e quatro ( $86 \%$ do total) na matriz extrato solúvel de soja.

Dentro dos critérios estabelecidos a validação do método QuEChERS-acetato foi adequada, visto que contemplou mais de $80 \%$ das substâncias selecionadas para o estudo. Em relação aos parâmetros de exatidão e precisão, a presença da matriz soja prejudicou a avaliação de um maior número de substâncias que na matriz extrato solúvel de soja. Também no parâmetro efeito matriz este foi observado em um maior número de substâncias na matriz soja em relação à matriz extrato solúvel de soja. Para as substâncias com resultados insatisfatórios nos parâmetros estudados modificações deverão ser feitas para adequação do método à análise destas substâncias.

O processo de validação da análise multirresíduo de agrotóxicos na matriz soja e extrato solúvel de soja é uma ferramenta a ser utilizada para atender aos limites definidos na legislação nacional e podendo ser aplicável a um maior número possível de substâncias em laboratórios que visam o monitoramento desses tipos de produtos alimentícios. Este processo proporciona subsídios aos órgãos regulatórios para verificar o uso dessas substâncias na prática agrícola e definir ações para controle e prevenção de possíveis danos à saúde da população consumidora dos alimentos produzidos.

\section{CONCLUSÃO}

No presente contexto, o desenvolvimento e otimização dos parâmetros da técnica analítica selecionada para o presente estudo demonstraram ser adequados para o método proposto dentro dos padrões internacionais de qualidade. Os requisitos mínimos de validação analítica foram alcançados nas duas matrizes avaliadas. A aplicação do método QuEChERS-acetato nas matrizes soja e extrato solúvel de soja representam um avanço das análises multirresiduais aplicado no laboratório para cerca de cento e vinte e duas substâncias validadas na matriz soja e cento e vinte e quatro na matriz extrato solúvel de soja.

O presente trabalho possibilitou a validação deste método para aplicação nas matrizes soja e extrato solúvel de soja com a associação da técnica de cromatografia líquida acoplada à espectrometria de massas sequencial permitindo o aumento do número de substâncias monitoradas em apenas uma análise.

\section{REFERÊNCIAS}

1. Ministério da Agricultura Pecuária e Abastecimento - MAPA. Soja. Disponível em: [http://www.agricultura.gov.br/vegetal/ culturas/soja].

2. Empresa Brasileira de Pesquisa Agropecuária Soja - EMBRAPA SOJA. Dados Econômicos da Soja. Disponível em: [http://www. cnpso.embrapa.br/index.php?op_page=294\&cod_pai=17].

3. Instituto Brasileiro de Geografia e Estatística - IBGE. Levantamento Sistemático da Produção Agrícola. Disponível em:[http://www.ibge.gov.br/home/estatistica/indicadores/ agropecuaria/lspa/lspa_201205_7.shtm]

4. Berno LI, Guimarães-Lopes TG, Canniatti-Brazaca SG. Avaliação da composição centesimal, digestibilidade e atividade inibitória de tripsina em produtos derivados de soja (glycine max). Alim Nutr.2007;18(3):277-82.

5. Anderson JW, Smith BM, Washnock CS. Cardiovascular and renal benefits of dry bean and soybean intake. Am J Clin Nutr.1999;70(supl):464S-74S.

6. Agência Nacional de Vigilância Sanitária - ANVISA. Monografias Autorizadas. Disponível em: [http://portal.anvisa.gov.br/wps/ portal/anvisa/home/agrotoxicotoxicologia].

7. Sobreira AEG, Adissi PJ. Agrotóxicos: falsas premissas e debates. Ciênc Saúde Coletiva.2003;8(4):985-90.

8. Pizzutti IR, De KoK A, Zanella R, Adaime MB, Hiemstra $\mathrm{M}$, Wickert $\mathrm{C}$ et al. Method validation for the analysis of 169 pesticides in soya grain, without clean up, by liquid chromatography-tandem mass spetrometry using positive and negative electrospray ionization. J Chromatogr A.2007;1142:123-36.

9. Anastassiades M., Lehotay SL, Stajnbaher D, Schenck FJ. Fast and Easy ultiresidue method employing acetonitrile extraction/ partitioning and "Dispersive Solid-Phase Extraction" for the determination of pesticide residues in produce. J AOAC Int.2003;86(2): 412-31.

10. Prestes OD, Friggi CA, Adaime MB, Zanella R. QUECHERS Um método moderno de preparo de pesticidas em alimentos por métodos cromatográficos acoplados à espectrometria de massas. Quim Nova.2009;32(6):1620-34. 
11. Pizzutti IR, De KoK A, Hiemstra M, Wickert C, Prestes OD. Method validation and comparison of acetonitrile extraction for the analysis of 169 pesticides in soya grain by liquid chromatography-tandem mass spetrometry. J Chromatogr A.2009;1216:4539-52.

12. Instituto Nacional de Metrologia, Qualidade e Tecnologia - INMETRO (Rio de Janeiro - Brasil). Orientações sobre Validação de Métodos de Ensaios Químicos: DOQ-CGCRE-008, rev. 01. Rio de Janeiro, 2003.

13. Instituto Nacional de Metrologia, Qualidade e Tecnologia INMETRO (Rio de Janeiro - Brasil). Orientação sobre Validação de Métodos Analíticos: DOQ-CGCRE-008, rev. 04. Rio de Janeiro, 2011.

14. Bazilio FS, Bonfim MVJ, Almeida RJ, Abrantes SMP. Uso de planilha eletrônica na verificação da adequação de curva analítica ao modelo linear. Analytica.2012;59:60-7.

15. Ribani M, Bottoli, CBG, Collins CH, Jardim ICSF. Validação em métodos cromatográficos e eletroforéticos. Quim Nova.2004;27(5):771-80.

16. Souza SVC, Junqueira RG. A procedure to assess linearity by ordinary least squares method. Anal Chim Acta.2005;552(1-2):25-35.

17. Thompson M, Ellison SLR, Wood R. Harmonized guidelines for a single-laboratory validation of methods of analysis. Pure Appl Chem.2002;74:835-55.

18. Dg-Sanco. Method Validation and Quality Control Procedures for Pesticide Residues Analysis in Food and Feed. Document No. SANCO/12495/2011 Disponível em: [http://ec.europa.eu/food/ plant/protection/resources/qualcontrol_en.pdf].

19. Souza SVC. Procedimento para validação intralaboratorial de métodos de ensaio: delineamento e aplicabilidade em análises de alimentos [tese de doutorado]. Belo Horizonte (MG): Universidade Federal de Minas Gerais, 2007.

20. Cardoso MHWM, Gouvêa AV, Nóbrega AW, Abrantes SMP. Validação de método para determinação de resíduos de agrotóxicos em tomate: uma experiência laboratorial. Ciênc Tecnol Aliment.2010;30(supl.1):63-72.

21. Prestes OD, Adaime MB, Zanella R. QuEChERS: possibilidades e tendências no preparo de amostra para determinação multirresíduo de pesticidas em alimentos. Scient Chromatogr.2011;3(1):51-64.

22. Hiemstra M, De Kok A. Comprehensive multi-residue method for the target analysis of pesticides in crops using liquid chromatography-tandem mass spectrometry. J Chromatogr A.2007;1154:3-25.

23. Pizzutti IR, Vreuls RJJ, De Kok A, Roehrs R, Martel S, Friggi CA et al. Design of a compressed air modulator to be used in comprehensive multidimensional gas chromatography and its application in the determination of pesticide residues in grapes. J Chromatogr A.2009;1216:3305-11.

24. Kovalczuk T, Lacina O, Jech M, Poustka J, Hajslova J. Novel approach to fast determination of multiple pesticide residues using ultra-performance liquid chromatographytandem mass spectrometry (UPLC-MS/MS). Food Addit Contam.2008;25(4):444-57.

25. Banerjee K, Oulkar DP, Dasgupta S, Patil SB, Patil SH, Savant R et al. Validation and uncertainty analysis of a multi-residue method for pesticides ingrapes using ethyl acetate extraction and liquid chromatography-tandem mass spectrometry. J Chromatogr A.2007;1173:98-109.
26. Leandro CC, Hancock P, Fussell RJ, Keely BJ. Comparison of ultra-performance liquid chromatography and highperformance liquid chromatography for the determination of priority pesticides in baby foods by tandem quadrupole mass spectrometry. J Chromatogr A.2006;1103:94-101.

27. Pareja L, Cesio V, Heinzen H, Fernandez-Alba AR. Evaluation of various QuEChERS based methods for the analysis of herbicides and other commonly used pesticides in polished rice by LC-MS/ MS. Talanta.2011;83(5):1613-22.

28. Mastovska K, Dorweiler KJ, Lehotay SJ, Wegscheid JS, Szpylka KA. Pesticide multiresidue analysis in cereal grains using modified Quechers method combined with automated direct sample introduction GC-TOFMS and UPLC-MS/MS techniches. J Agric Food Chem.2010;58(10):5959-72.

29. Romero-Gonzalez R, Frenich AG, Vidal JM. Multiresidue method for fast determination of pesticides in fruit juices by ultraperformance liquid chromatography coupled to tandem mass spectrometry. Talanta.2008;76:211-25.

30. Mol HGJ, Plaza-Bolanos P, Zomer P, Rijk TC, Stolker AAM, Mulder PPJ. Toward a generic extraction method for simultaneos determination of pesticides, mycotoxins, plant toxins, and veterinary drugs in feed and food matrixes. Anal Chem.2008;80:9450-9.

31. Durden DA. Positive and negative electrospray LC-MS-MS methods for quantitation of the antiparasitic endectocide drugs, abamectin, doramectin, emamectin, eprinomectin, ivermectin, moxidectin and selamectin in milk. J Chromatogr B.2007;850:134-46.

32. Agilent Technologies (Santa Clara - EUA). Multiresidue Analysis of 301 Pesticides in Food Samples by LC/Triple Quadrupole Mass Spectrometry: 5989-8614EN. Santa Clara, 2008.

33. Pang G, Cao Y, Zhang J, Fan C, Liu Y, Li X et al. Validation study on 660 pesticide residues in animal tissues by gel permeation chromatography cleanup/gas chromatographymass spectrometry and liquid chromatography-tandem mass spectrometry. J Chromatogr A.2006;1125(1):1-3

34. Waters (Milford - EUA). A rapid method for the screening and confirmation of over 400 pesticide residues in food: 720002628EN. Milford, 2012.

35. Fillâtre Y, Rondeau D, Jadas-Hécart A, Communal PY. Advantages of the scheduled selected reaction monitoring algorithm in liquid chromatography/electrospray ionization tandem mass spectrometry multi-residue analysis of 242 pesticides: a comparative approach with classical selected reaction monitoring mode. Rapid Commun Mass Spectrom.2010;24:2453-61.

36. Sack C, Smoker M, Chamkasem N, Thompsom R, Satterfield $\mathrm{G}$, Masse C et al. Collaborative Validation of the QuEChERS Procedure for the Determination of Pesticides in Food by LCMS/MS. J Agric Food Chem.2011;59:6383-411.

37. Alder L. Residue analysis of 500 high priority pesticides: better by GC-MS or LC-MS/MS. Mass Spectrom Rev.2006;25:838-65.

38. Agilent Technologies (Santa Clara - EUA). Multiresidue Analysis of 301 Pesticides in Food Samples by LC/Triple Quadrupole Mass Spectrometry: 5991-1183EN. Santa Clara, 2012.

39. Chen G, Cao P, Liu R. A multi-residue method for fast determination of pesticides in tea by ultra performance liquid chromatography-electrospray tendem mass spectrometry combined with modified QuEChERS sample preparation procedure. Food Chem.2011;125:1406-11. 